\title{
The potential roles of vesicle-enclosed miRNAs in communication between macrophages and cancer cells in tumor microenvironment
}

\author{
M. KORABECNA ${ }^{1, *}$, L. KOUTOVA², P. TESAROVA ${ }^{3}$ \\ ${ }^{1}$ Department of Biology and Medical Genetics, First Faculty of Medicine, Charles University in Prague and General Faculty Hospital in Prague, \\ Albertov 4, 12800 Prague, Czech Republic; ${ }^{2}$ Department of Hematooncology, Charles University Hospital in Pilsen, Alej Svobody 80,30460 \\ Pilsen, Czech Republic; ${ }^{3}$ Department of Oncology, First Faculty of Medicine, Charles University in Prague and General Faculty Hospital in Prague, \\ U Nemocnice 2, 12808 Prague, Czech Republic
}

${ }^{*}$ Correspondence: marie.korabecna@lf1.cuni.cz

Received August 4, 2016 / Accepted December 4, 2016

\begin{abstract}
Functional microRNA (miRNA) molecules are transported in extracellular vesicles among tumor cells and cells of the immune system. Macrophages as integral components of tumor microenvironment are known as potential contributors to tumor growth and progression. We searched for studies which could provide a direct link between the particular miRNAs transported between cancer cells and macrophages and experimental evidence of subsequent alterations in biological functions of target cells. The validated targets of such microRNAs were found using miRWalk database. These targets were further subjected to analysis by DAVID (Database for Annotation, Visualization and Integrated Discovery) to find the most prominent cellular events that could be potentially regulated in macrophages by miRNAs originated from cancer cells and vice versa. We found that the 5 miRNAs (let-7b, miR-21, miR-29a, miR-222-3p, miR-451) derived from cancer cells may together regulate 2304 target genes in macrophages. The genes involved in regulation of apoptosis, regulation of gene expression and protein transport were significantly overrepresented in this set. Four of the five sets of target genes for these individual miRNAs overlap in MYC oncogene. MYC dependent transcriptional program is responsible for cell cycle entry and regulates the inflammatory response in macrophages.

Both miRNAs for which the functional transports from macrophages to cancer cells were experimental proven (miR-223, miR-142-3p) target total 684 genes including some well-known tumor suppressors like TP53 or APC. Suppression of tumor suppressor genes by miRNAs derived from macrophages may eventually contribute to cancer cell proliferation.

Due to the complexity of tumor microenvironment, the altered expression profiles of its components affected by miRNA uptake from extracellular vesicles could contribute to the outcome of carcinogenesis therefore the vesicular transport of miRNAs should be studied more extensively in this context.
\end{abstract}

Key words: microRNA, extracellular vesicles, cancer microenvironment, macrophage, cancer cell

In the last years, enormous amount of work had been done to understand the biological functions of microRNA (miRNA). MicroRNAs were recognized as powerful post-transcriptional regulators, it has been estimated that one third of human genome may be regulated by them $[1,2]$. The mature miRNAs act in the post-transcriptional regulation of gene expression. The miRNA molecule is perfectly or partially complementary to its target mRNA. The degree of complementarity is crucial for the fate of the target mRNA. The perfect or nearly perfect complementarity, which is very rare in mammals, leads to a site-specific cleavage of the target mRNA and it is generally called RNA interference. Two more possibilities can happen to more mismatched mRNA - either the enhanced degradation of the target mRNA or inhibition of its translation - both representing a non-cleavage repression [3].

In this article, we focused on summarization and bioinformatic evaluation of existing experimental evidence for vesicular transport of particular functional microRNA molecules among tumor cells and macrophages in the context of carcinogenesis. Macrophages were selected not only with regards to their availability for experiments and to their frequent involvement in experimental studies, but mainly as very important components of tumor microenvironment and having the potential to utilize some mechanisms included in 
the process of wound healing for tumor growth and progression [4]. With regards to macrophage phenotype, the most widely accepted classification describes two basic types: classical (M1) versus alternative (M2). The response of M1 macrophages is pro-inflammatory characterized by the production of cytotoxic factors, increased rates of phagocytosis, and enhanced antigen presentation on the cell surface. M2 macrophages develop as part of the wound healing program. They antagonize inflammation, secrete angiogenesis promoting factors and produce enzymes remodeling the surrounding extracellular matrix. It is well known that chronic inflammation enhances the risk of cancer development. Tissue-resident macrophages are important for maintaining a balanced state referred to as inflammatory homeostasis [4]. Phenotypes of tumor associated macrophages (TAM) may include the combination of markers of both M1 and M2 types depending on the stage of carcinogenesis [4]. We searched for such studies which provide the direct experimental evidence that the uptake of a miRNA accepted by macrophages in the form of extracellular vesicles derived from cancer cells is able to alter biological functions of this target cells. We tried also to follow the opposite direction of vesicular miRNA transport - from macrophages to cancer cells.
We focused only on studies [5-15] documenting the altered cellular functions of recipient cells due to the uptake of specific miRNA molecules included in extracellular vesicles. The results of the studies fulfilling this criterion are summarized in Figure 1 and described in the next paragraphs. Using exosomes isolated from lung carcinoma cell line, the data were obtained that indicated that miR-29a and miR-21 secreted in exosomes could bind to endosomal TLR8 in macrophages at the tumor interface and induce TLR8-mediated activation of $\mathrm{NF}-\kappa \mathrm{B}$ and NF- $\kappa \mathrm{B}-$ mediated secretion of pro-inflammatory cytokines TNF- $\alpha$ and IL-6 [5].

The study by Li et al. [6] demonstrated that macrophages acquired tumor-derived microRNA let- $7 \mathrm{~b}$ which was contained in vesicles and which attenuated tumor-associated inflammation by down regulation of cytokine IL-6 production [6].

Salama et al. [7] studied the effect of pancreatic beta-cell specific miRNAs transfection on macrophages and dendritic cells in mice. The in vitro research revealed the transport of miR-29 in exosomes from pancreatic beta-cells into macrophages and the dose dependent increase of TNF-a secretion by these recipient cells. This study demonstrated that the exosomal miRNA transfer targeted to cells of innate immunity is not limited to cancer cells as miRNA donors.

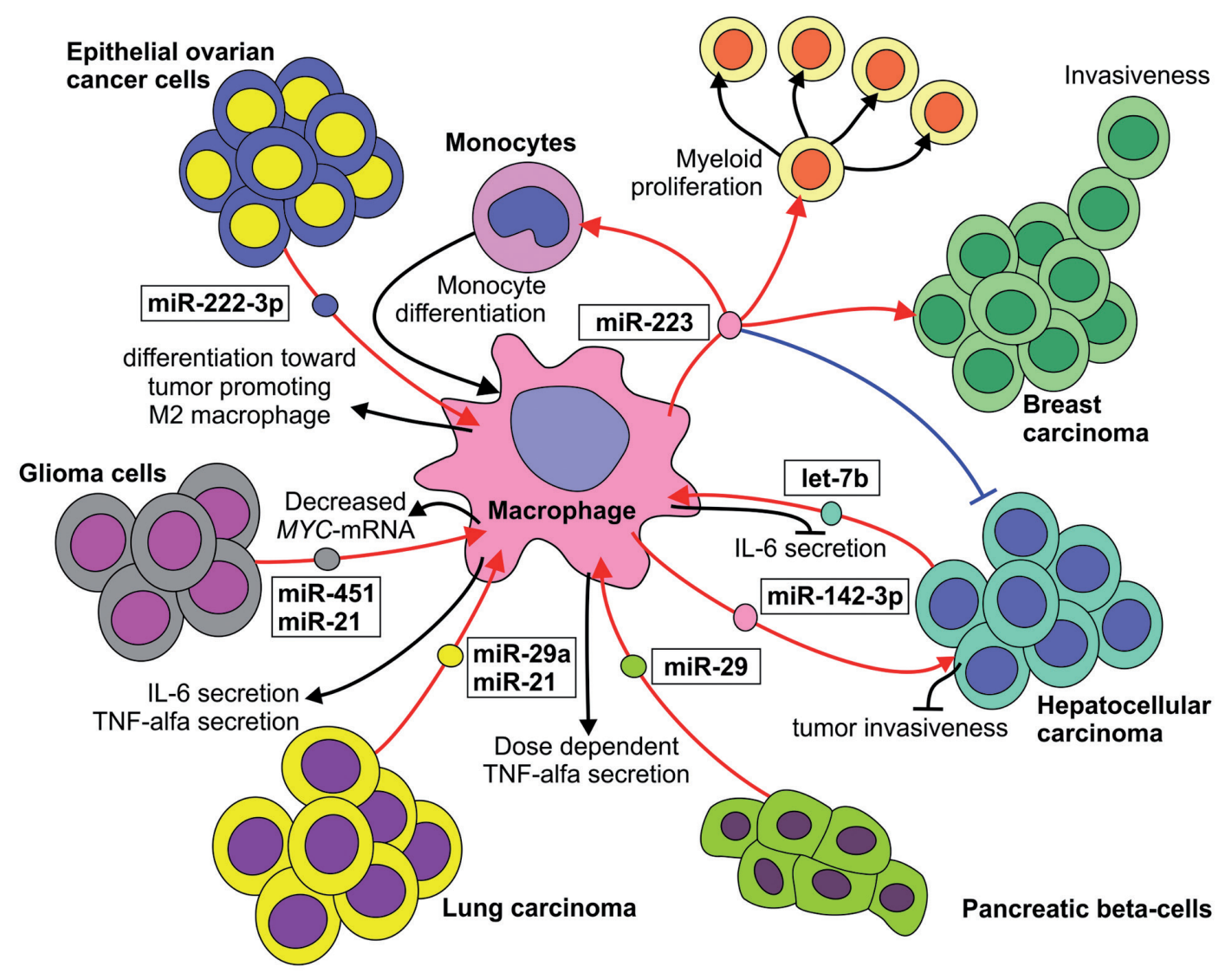

Figure 1. MicroRNAs transported via extracellular vesicles among macrophages and tumor cells. Details are discussed in the text together with references. 
A miRNA molecule produced by IL-4 activated macrophages - miR-223 - was transported inside exosomes into breast cancer cells in co-cultivation experiments. The increased invasiveness of co-cultivated cancer cells was observed. The application of $\mathrm{mR}-223$ antisense oligonucleotide diminished this effect [8]. Human glioblastoma-derived extracellular vesicles transported high content of miR-451/miR-21 to microglia and monocytes/macrophages from tumor-bearing brains. The reduced levels of MYC mRNA were observed in these target cells [9]. Epithelial ovarian cancer-derived exosomes having high content of miR-222-3p activated macrophages to a tumor-associated macrophage (TAM)- which could facilitate the progression of cancer. Overexpression of miR-222-3p in macrophages induced their polarization to the M2 phenotype [10]. The miR-223 had been found in macrophage-derived microvesicles as having the highest expression among 186 other miRNAs contained in these vesicles. The miR-223 transferred in such microvesicles was functional in the recipient cells - monocytes. MiR-223 was associated with the induction of monocyte to macrophage differentiation [11]. The miR-223 was also recognized as the most abundant miRNA in peripheral blood microvesicles. It probably plays a variety of important regulatory roles as a systemic homeostatic factor [12]. It had been demonstrated that miR-223 obtained in the form of vesicles from macrophages can stimulate the myeloid proliferation [13].

The role of exosomal miR-223 in cancer suppression has been studied by Aucher et al. [14]. The exosomes containing miR-223 and miR-142 were released by monocyte-derived macrophages and transported to co-cultivated hepato-carcinoma cells in a manner that required intercellular contact and involved gap junctions. Such a transfer inhibited proliferation of cancerous cells [14].

The tumor suppressing functions of miR-142-3p delivered in microvesicles from tumor associated macrophages to hepatocellular tumor cells was demonstrated in mice model. Animals having tumors were treated with propofol which is known for its anti-tumor effects. Down-regulation of the expression miR-142-3p using its inhibitor was able to reverse the effect of propofol on hepatocarcinoma cells [15].

Although the microenvironment in various types of tumors differs remarkably, macrophages are present here regularly. Bert Vogelstein [16] determined 12 cellular pathways which are affected in the process of carcinogenesis independently on tumor type. Due to this general aspect of carcinogenesis and due to the wide spectrum of targets of each miRNA, we could evaluate the pooled targets of miRNAs fulfilling our above described selection criteria.

Using MirWalk database [17], we searched for validated targets of miRNAs for which the functional vesicular transport between cancer cells and macrophages was experimentally proven. The validated target genes were further analyzed using the Database for Annotation, Visualization and Integrated Discovery (DAVID) $[18,19]$. Overlapping gene sets playing potential roles in tuning of immune system performance were found: In the sets of target genes in macrophages, we found $M Y C$ as the prominent target of four out of the five analyzed miRNAs. The role of MYC expression for the determination of macrophage phenotype had been reported [20]. The sets of target genes in cancer cells contain numerous tumor suppressor genes. This fact highlights the potential tumor-promoting roles of macrophages targeted by tumor-derived vesicles containing the analyzed miRNAs.

We provide the first preview of potential pathways and regulatory events which may be affected in consequence of vesicular miRNA exchange between macrophages and cancer cells.

\section{Material and methods}

We analyzed the results of studies published till the June 2016 to find experimental evidence of functional transport of particular miRNA molecules in extracellular vesicles between cancer cells and macrophages. We included only such studies which assign the altered biological functions of target cells to the uptake of particular miRNA due to the employment of an existing method for this miRNA inhibition in their experimental workflows.

After selection of studies (cited in Introduction) fulfilling this criterion we searched using miRWalk database for the validated targets of selected miRNAs. These target genes were further analyzed using the Database for Annotation, Visualization and Integrated Discovery (DAVID) $[18,19]$ by the functional annotation clustering with the medium classification stringency and with calculations of Benjamini corrections and false discovery rates (FDR) to find regulatory pathways which may be significantly affected due to the uptake of miRNAs from extracellular vesicles. The Venn diagrams were constructed using the web based tool (http://bioinformatics. psb.ugent.be/webtools/Venn).

\section{Results}

Using MirWalk database [17], we searched for validated targets of miRNAs for which the functional vesicular transports from cancer cells to macrophages were proven. The numbers of detected validated targets for let-7b, miR-21, miR-29a, miR-222-3p and miR-451 and the extent of overlaps among these individual gene sets are documented in Figure 2. All target genes for each individual miRNA are listed in Supplementary Table 1. All target genes were further analyzed using DAVID $[18,19]$ as described in Methods. Table 1 demonstrates the results of this analysis. Overrepresented gene sets playing roles in crucial cellular pathways like regulation of apoptosis, regulation of transcription and protein transport were found.

Four out of the five sets of validated target genes for individual miRNAs overlap in the oncogene MYC (myelocytomatosis viral oncogene) (Figure 2). The lists of genes targeted by three out of five selected miRNAs are included also in Figure 2. 


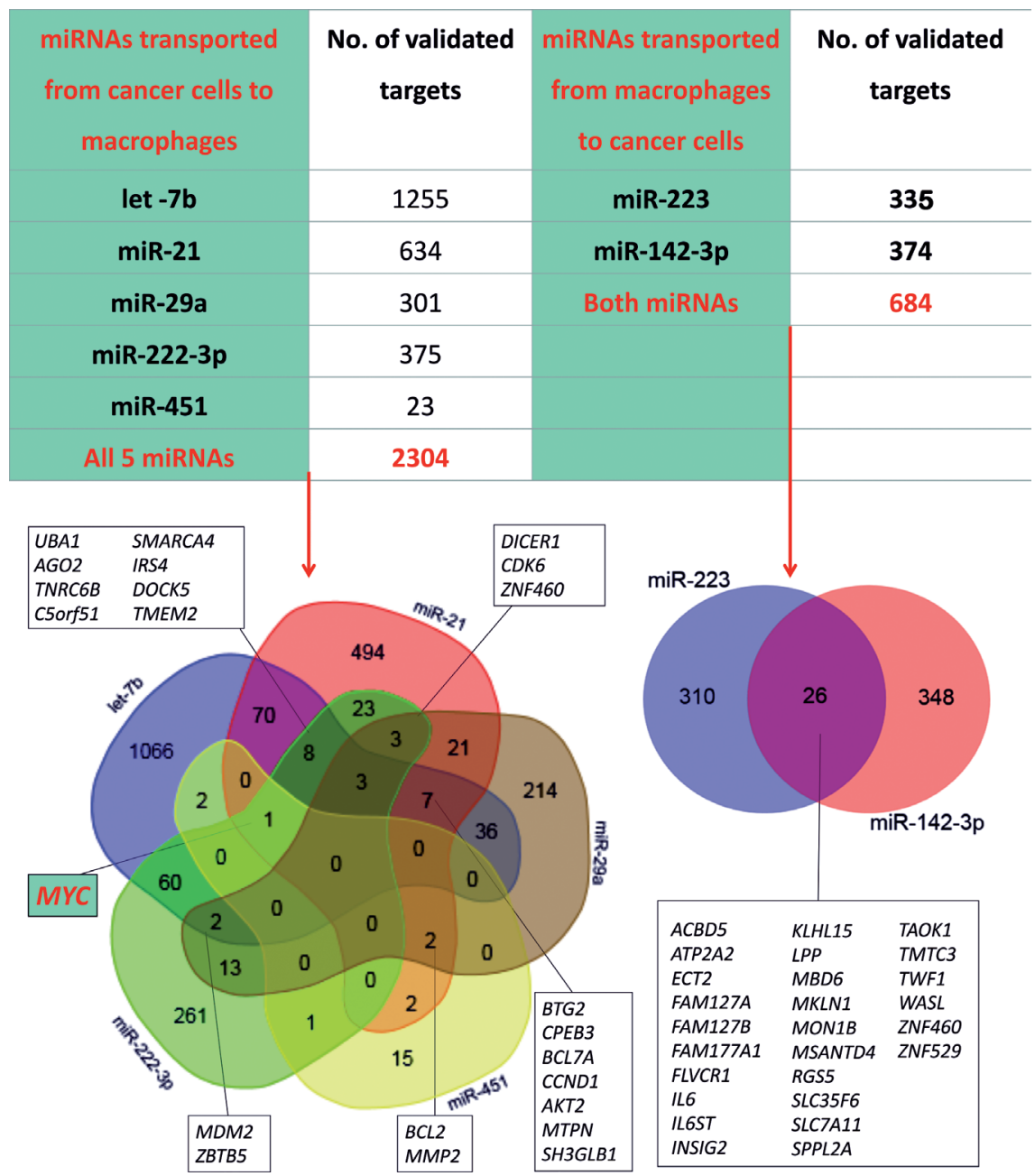

Figure 2. Numbers of validated targets for each analyzed miRNA and extent of overlaps among individual gene sets.

We searched also for validated targets of miRNAs for which the functional vesicular transport from macrophages to cancer cells was proven. We found 335 validated targets for miR-223a and 374 validated targets for miR-142-3p. The sets of target genes for each of these miRNA are listed in Supplementary Table 2. The results of DAVID analysis of all these targets are summarized in Table 2. The target genes grouped under the term "Pathways in cancer" and their functions in cancer related regulatory pathways are schematically represented in Figure 3. The validated targets of miR-223a and miR-142-3p overlap in 26 genes (Figure 2). The DAVID analysis of these 26 target genes did not revealed any overrepresented cluster of functionally associated genes.

\section{Discussion}

It is necessary to keep in mind that our analysis of validated miRNA target genes and their functional clustering provides
Table 1. Top terms identified by DAVID for all targets of miRNAs transported in vesicles from cancer cells into macrophages. p-values were subjected to Benjamini corrections, FDR - false discovery rate

\begin{tabular}{lccc}
\hline Term & $\begin{array}{c}\text { No. of } \\
\text { genes }\end{array}$ & p-value & FDR \\
\hline Membrane -enclosed lumen & 399 & $5.2 \times 10^{-35}$ & $1.1 \times 10^{-34}$ \\
Non-membrane bound organelle & 449 & $2.5 \times 10^{-18}$ & $3.8 \times 10^{-17}$ \\
Nucleus & 750 & $1.1 \times 10^{-41}$ & $6.9 \times 10^{-41}$ \\
Regulation of transcription & 435 & $3.4 \times 10^{-7}$ & $4.3 \times 10^{-6}$ \\
Nucleotide binding & 313 & $3.0 \times 10^{-18}$ & $3.3 \times 10^{-17}$ \\
Cell cycle & 177 & $1.2 \times 10^{-10}$ & $5.3 \times 10^{-11}$ \\
Negative regulation of gene expression & 125 & $7.5 \times 10^{-10}$ & $1.3 \times 10^{-9}$ \\
Protein transport & 103 & $1.2 \times 10^{-8}$ & $4.6 \times 10^{-7}$ \\
Regulation of cell death & 167 & $2.6 \times 10^{-7}$ & $2.7 \times 10^{-6}$ \\
Regulation of apoptosis & 164 & $3.1 \times 10^{-7}$ & $3.6 \times 10^{-6}$ \\
Pathways in cancer & 92 & $4.4 \times 10^{-10}$ & $3.0 \times 10^{-9}$ \\
\hline
\end{tabular}


Table 2. Top terms identified by DAVID for all targets of miRNAs transported in vesicles from macrophages into cancer cells. p-values were subjected to Benjamini corrections, FDR - false discovery rate

\begin{tabular}{lccc}
\hline Term & No. of genes & p-value & FDR \\
\hline Regulation of transcription & 143 & $7.7 \times 10^{-4}$ & $2.5 \times 10^{-3}$ \\
Nucleus & 205 & $1.1 \times 10^{-4}$ & $1.3 \times 10^{-3}$ \\
Pathways in cancer & 34 & $6.5 \times 10^{-4}$ & $5.2 \times 10^{-3}$ \\
& (Details in Figure 3) & \\
\hline
\end{tabular}

only the first preview of potential pathways and regulatory events which may be affected in macrophages and cancer cells as a consequence of uptake of miRNA molecules from vesicles exchanged between these cells.

We highlighted the role of the gene $M Y C$ as a prominent target of miRNAs targeted from cancer cells to macrophages (Figure 2). Recently, the central role of this gene in macrophage phenotype determination has been gradually emerging. Liu et al. [20] found that MYC expression plays a major role in regulating proliferation-associated metabolic programs in macrophages. Pro-inflammatory stimuli suppress MYC and cell proliferation and start a hypoxiainducible factor alpha (HIF1a)-dependent transcriptional program which is responsible for heightened glycolysis. This switch may regulate the robust bioenergetic support for inflammatory response [20].
We also found the gene DICER 1 as a target gene of three out of five tumor-derived miRNAs transported to macrophages (Figure 2). The gene codes for a cytoplasmic endonuclease (RNase III enzyme Dicer) which is crucial for the process of miRNA processing itself $[21,22]$.

Using DAVID analysis, we found the overrepresented group of 34 genes involved in "Pathways in cancer" in the set of genes targeted in cancer cells by macrophage-derived miRNAs. This group of genes (Figure 3) deserves especial attention because it contains both oncogenes and tumor suppressor genes and therefore it may well illustrate the potential dual role of tumor associated macrophages which is highly dependent on actual state of complex tumor microenvironment.

The results documenting the communication among cancer cells and immune cells via vesicles represent the theoretical platform for the development of therapeutic approaches based on the delivery of specific miRNA molecules inside artificial exosomes to carefully selected subset of target cells to activate the immune response against tumors or to avoid tumor angiogenesis. The first attempts streaming to application of this concept had been already made $[23,24]$.

Despite the first promising results, an enormous effort must be made to overcome all the difficulties associated with the complexity of regulatory events operating on tumor-immune system interface with the goal to develop a safe personalized type of anti-tumor therapy based on vesicular delivery of miRNAs to target cells.

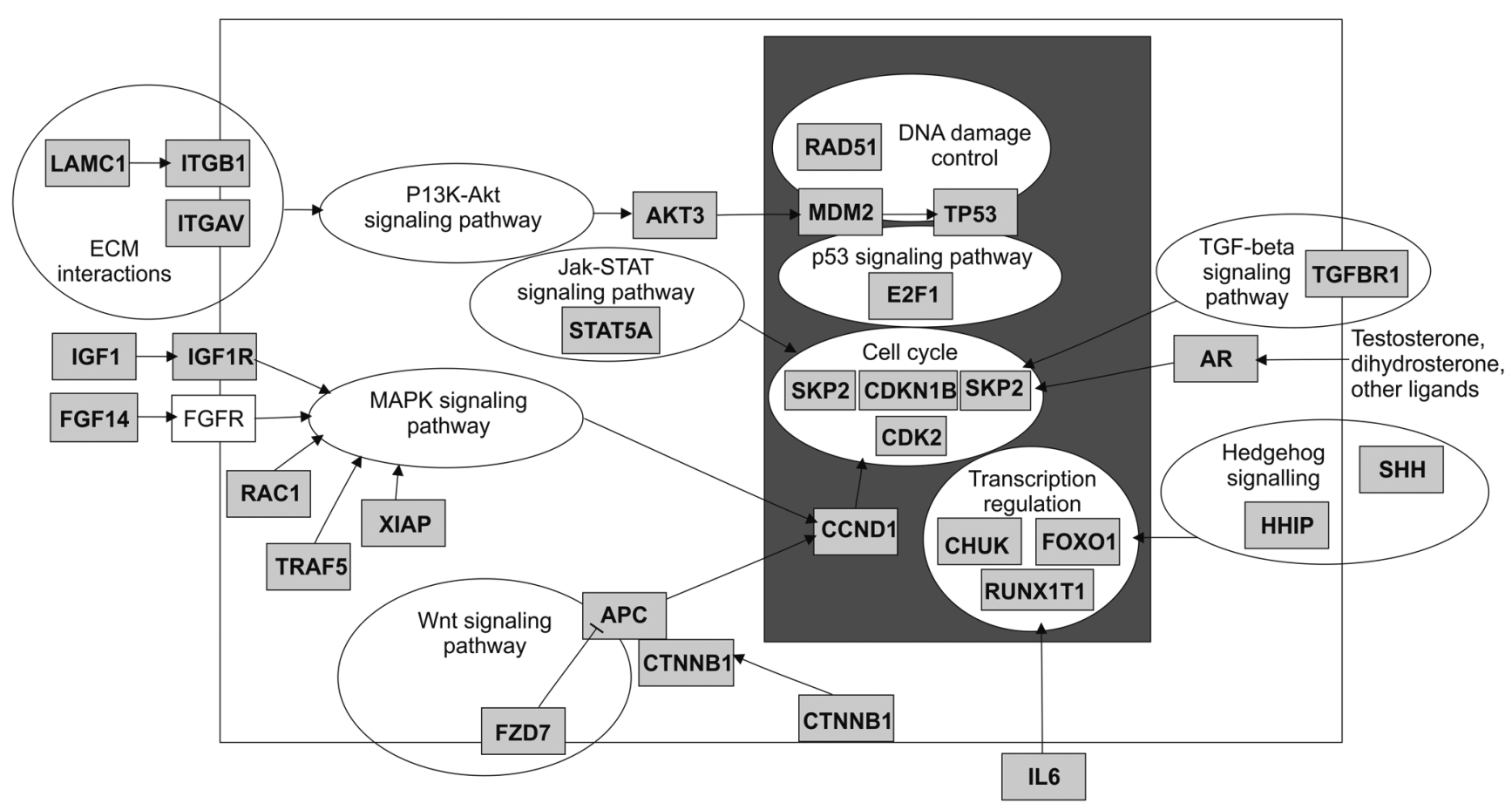

Figure 3. Target genes of miR-233 and/or miR-142-3p included in "Pathways in cancer" are depicted according to http://www.genome.jp/kegg-bin/ show_pathway?hsa05200 with exception of genes CCDC6, HSP90B1,PIK3CG and TFG. 
Supplementary information is available in the online version of the paper.

Acknowledgements: M.K. and P.T. were supported by the grant no. PRVOUK P25/LF1/2 of the Ministry of Education, Youth and Sport of the Czech Republic, M.K. was supported also by the grants RVO-VFN 64165 of the Ministry of Health of the Czech Republic.

\section{References}

[1] LEWIS BP, BURGE CB, BARTEL DP. Conserved seed pairing, often flanked by adenosines, indicates that thousands of human genes are microRNA targets. Cell 2005; 120: 15-20. https://doi.org/10.1016/j.cell.2004.12.035

[2] BENTWICH I. A postulated role for microRNA in cellular differentiation. FASEB J 2005; 19: 875-879. https://doi. org/10.1096/fi.04-3609hyp

[3] HA M, KIM VN. Regulation of microRNA biogenesis. Nat Rev Mol Cell Biol 2014; 15: 509-524. https://doi.org/10.1038/ $\underline{\operatorname{nrm} 3838}$

[4] BRADY NJ, CHUNTOVA P, SCHWERTFEGER KL. Macrophages: Regulators of the inflammatory microenvironment during mammary gland development and breast cancer. Mediators Inflamm 2016; 2016: 4549676. https://doi. org/10.1155/2016/4549676

[5] FABBRI M, PAONE A, CALORE F, GALLI R, GAUDIO E et al. MicroRNAs bind to Toll-like receptors to induce prometastatic inflammatory response. Proc Natl Acad Sci USA 2012; 109: E2110-E2116. https://doi.org/10.1073/pnas.1209414109

[6] LI D, JIA H, ZHANG H, LV M, LIU J et al. TLR4 signaling induces the release of microparticles by tumor cells that regulate inflammatory cytokine IL- 6 of macrophages via microRNA let-7b. Oncoimmunology 2012; 1: 687-693. https:// doi.org/10.4161/onci.19854

[7] SALAMA A, FICHOU N, ALLARD M, DUBREIL L, DE BEAUREPAIRE L et al. MicroRNA-29b modulates innate and antigen-specific immune responses in mouse models of autoimmunity. PLoS One 2014; 9: e106153. https://doi. org/10.1371/journal.pone.0106153

[8] YANG M, CHEN J, SU F, YU B, SU F et al. Microvesicles secreted by macrophages shuttle invasion-potentiating microRNAs into breast cancer cells. Mol Cancer 2011; 10: 117. https://doi.org/10.1186/1476-4598-10-117

[9] VAN DER VOS KE, ABELS ER, ZHANG X, LAI C, CARRIZOSA E et al. Directly visualized glioblastoma-derived extracellular vesicles transfer RNA to microglia/macrophages in the brain. Neuro Oncol 2016; 18: 58-69. https://doi. org/10.1093/neuonc/nov244

[10] YING X, WU Q, WU X, ZHU Q, WANG X et al. Epithelial ovarian cancer-secreted exosomal miR-222-3p induces polarization of tumor-associated macrophages. Oncotarget 2016; 7: 43076-43087.

[11] ISMAIL N, WANG Y, DAKHLALLAH D, MOLDOVAN L, AGARWAL K et al. Macrophage microvesicles induce macrophage differentiation and miR-223 transfer. Blood 2013; 121: 984-995. https://doi.org/10.1182/blood-2011-08-374793
[12] HANEKLAUS M, GERLIC M, O'NEILL LA, MASTERS SL. miR-223: infection, inflammation and cancer. J Intern Med 2013; 274: 215-226. https://doi.org/10.1111/joim.12099

[13] JOHNNIDIS JB, HARRIS MH, WHEELER RT, STEHLINGSUN S, LAM MH et al. Regulation of progenitor cell proliferation and granulocyte function by microRNA-223. Nature 2008; 451: 1125-1129. https://doi.org/10.1038/nature 06607

[14] AUCHER A, RUDNICKA D, DAVIS DM. MicroRNAs transfer from human macrophages to hepato-carcinoma cells and inhibit proliferation. J Immunol 2013; 191: 6250-6260. https:// doi.org/10.4049/jimmunol.1301728

[15] ZHANG J, SHAN WF, JIN TT, WU GQ, XIONG XX et al. Propofol exerts anti-hepatocellular carcinoma by microvesicle-mediated transfer of miR-142-3p from macrophage to cancer cells. J Transl Med 2014; 12: 279. https://doi. org/10.1186/s12967-014-0279-X

[16] VOGELSTEIN B, PAPADOPOULOS N, VELCULESCU VE, ZHOU S, DIAZ LA jr et al. Cancer genome landscapes. Science 2013; 339: 1546-1558. https://doi.org/10.1126/science. 1235122

117] DWEEP H, STICHT C, PANDEY P, GRETZ N. miRWalk-database: prediction of possible miRNA binding sites by „walking“the genes of three genomes. J Biomed Inform 2011; 44: 839-847. https://doi.org/10.1016/j.jbi.2011.05.002

]18] HUANG DW, SHERMAN BT, LEMPICKI RA. Bioinformatics enrichment tools: paths toward the comprehensive functional analysis of large gene lists. Nucleic Acids Res 2009; 37: 1-13. https://doi.org/10.1093/nar/gkn923

[19] HUANG DW, SHERMAN BT, LEMPICKI RA. Systematic and integrative analysis of large gene lists using DAVID bioinformatics resources. Nat Protoc 2009; 4: 44-57. https://doi. org/10.1038/nprot.2008.211

[20] LIU L, LU Y, MARTINEZ J, BI Y, LIAN G et al. Proinflammatory signal suppresses proliferation and shifts macrophage metabolism from Myc-dependent to HIF1alpha-dependent. Proc Natl Acad Sci USA 2016; 113: 1564-1569. https://doi. org/10.1073/pnas.1518000113

[21] GRISHOK A, PASQUINELLI AE, CONTE D, LI N, PARRISH $S$ et al. Genes and mechanisms related to RNA interference regulate expression of the small temporal RNAs that control C. elegans developmental timing. Cell 2001; 106: 23-34. https:// doi.org/10.1016/S0092-8674(01)00431-7

[22] KNIGHT SW, BASS BL. A role for the RNase III enzyme DCR-1 in RNA interference and germ line development in Caenorhabditis elegans. Science 2001; 293: 2269-2271. https:// doi.org/10.1126/science.1062039

[23] LIU Y, ZHAO L, LI D, YIN Y, ZHANG CY et al. Microvesicledelivery miR-150 promotes tumorigenesis by up-regulating VEGF, and the neutralization of miR-150 attenuate tumor development. Protein Cell 2013; 4: 932-941. https://doi. org/10.1007/s13238-013-3092-Z

[24] DE ROSA F, FANINI F, GUIDOBONI M, VANNINI I, AMADORI D et al. MicroRNAs and dendritic cell-based vaccination in melanoma patients. Melanoma Res 2014; 24: 181-189. https://doi.org/10.1097/CMR.0000000000000058 
Supplementary Table 1

Official Gene Symbol AARSD1

AASDHPPT

AATF

ABCB10

ABCB8

ABCC1

ABCC4

ABCF1

ABHD17C

ABL1

ABT1

ACACA

ACER2

ACOT9

ACP1

ACPP

ACSL1

ACTA1

ACTB

ACTG1

ACTN4

ACVR1

ACVR2B

ADCK2

ADCY1

ADH1B

ADH5

ADIPOR2

ADNP

ADRM1
Official Gene Symbol

Validated targets of hsa-miR-21:

$A B C D 3$

ACAT1

ACBD5

ACTR2

ADNP

AFTPH

AGAP1

AGBL3

AGGF1

AGO2

$\mathrm{AGO} 4$

AHSA2

AIM1

AKAP9

AKT2

ALMS1

AMMECR1L

ANKRD28

ANKRD46

ANP32A

AP1AR

AP3M1

APAF1

APC

APOLD1

APPL1

ARHGAP21

ARHGEF12

ARID4A

ARMCX3
Official Gene Symbol

Validated targets of hsa-miR-29a:

AAK1
ABCE1
ABL1
ADAMTS9
AG01
AHSA2
AIM1
AKT2
AMER1
AMFR
AMMECR1L
ASXL2
BACE1
BBC3
BCAP31
BCL2
BCL7A
BMPR1A
BTG2
C18orf32
C1QTNF6
C21orf91
CALCR
CALM3
CAND1
CASP8
CBX2
CBX6
CCDC117
CCDC14




$\begin{array}{cc}\text { AFAP1 } & \text { ARNTL } \\ \text { AFF4 } & \text { ARRDC2 } \\ \text { AGFG2 } & \text { ASRGL1 } \\ \text { AGL } & \text { ATAD2B } \\ \text { AGO1 } & \text { ATF2 } \\ \text { AGO2 } & \text { ATF7IP } \\ \text { AGO3 } & \text { ATMIN } \\ \text { AHCTF1 } & \text { ATP11B } \\ \text { AHCYL1 } & \text { ATP2B4 } \\ \text { AHCYL2 } & \text { ATRX } \\ \text { AHR } & \text { ATXN10 } \\ \text { AIDA } & \text { AUTS2 } \\ \text { AK4 } & \text { B3GALNT1 } \\ \text { AKAP8 } & \text { B3GNT5 } \\ \text { AKR1A1 } & \text { BASP1 } \\ \text { AKT2 } & \text { BAZ1B } \\ \text { ALDH1A3 } & \text { BCAT1 } \\ \text { ALDH7A1 } & \text { BCL2 } \\ \text { ALG10B } & \text { BCL6 } \\ \text { ALG3 } & \text { BCL7A } \\ \text { AMD1 } 10 \\ \text { AMPH } & \text { BDH2 } \\ \text { ANAPC1 } & \text { BMPR2 } \\ \text { ANAPC16 } & \text { BNIP2 } \\ \text { ANKRD17 } & \text { BOC } \\ \text { ANKRD46 } & \text { BRCA1 } \\ \text { ANKRD52 } & \text { BTBD3 } \\ \text { ANKZF1 } & \text { BTBD7 } \\ \text { ANP32E } & \text { BTG2 } \\ \text { ANTXR1 } & \text { BTN3A3 } \\ \text { ANXA8 } & \text { C10orf137 } \\ \text { ANXA8L1 } & \text { C15orf52 } \\ \text { ANXA8L2 } & \text { C20orf194 } \\ & \text { C2orf43 }\end{array}$

CCNA2

CCND1

CCND2

CCNT2

CCT4

CD276

CD93

CDC23

CDC42

CDC42SE1

CDC7

CDK2

CDK4

CDK6

CEACAM6

CFHR3

CLSPN

CMBL

CNBP

COL10A1

COL3A1

COL4A1

COL4A2

COL5A2

COLEC10

COMMD2

COX7A2L

CPEB3

CPEB4

CRKL

CSRNP2

CTC1

CTNNBIP1 


AP1S1
AP3M1
APPL1
APRT
AR
ARAP2
ARCN1
AREL1
ARFIP2
ARHGAP17
ARHGAP26
ARHGEF5
ARID1B
ARID3A
ARID3B
ARIH1
ARL15
ARL6IP1
ARL8B
ARPP19
ASB11
ASCC3
ASIC1
ASNA1
ASPA
ASPSCR1
ATAD3B
ATE1
ATG12
ATG4B
ATG9A
ATOX1
ATP1A1

$\begin{array}{cc}\text { C4orf32 } & \text { CXCR2 } \\ \text { C5orf51 } & \text { CYR61 } \\ \text { CALD1 } & \text { DAG1 } \\ \text { CALM1 } & \text { DCTN6 } \\ \text { CAPRIN1 } & \text { DDX6 } \\ \text { CASC5 } & \text { DHX33 } \\ \text { CBX4 } & \text { DIABLO } \\ \text { CCDC14 } & \text { DICER1 } \\ \text { CCDC34 } & \text { DKK1 } \\ \text { CCL1 } & \text { DNMT1 } \\ \text { CCL20 } & \text { DNMT3A } \\ \text { CCND1 } & \text { DNMT3B } \\ \text { CCNG1 } & \text { DPF3 } \\ \text { CCR1 } & \text { DSC2 } \\ \text { CCR7 } & \text { DUSP2 } \\ \text { CD47 } & \text { DYNLT1 } \\ \text { CDC25A } & \text { EIF3E } \\ \text { CDC42 } & \text { ELMSAN1 } \\ \text { CDK19 } & \text { EMP1 } \\ \text { CDK2AP1 } & \text { ENPP2 } \\ \text { CDK6 } & \text { ENTPD1 } \\ \text { CELF1 } & \text { EPHX2 } \\ \text { CENPQ } & \text { FAM102B } \\ \text { CEP152 } & \text { FAM109B } \\ \text { CEP97 } & \text { FAM133A } \\ \text { CERS6 } & \text { FAM193A } \\ \text { CKAP5 } & \text { FAM208B } \\ \text { CLASP1 } & \text { FAM71F2 } \\ \text { CLCN5 } & \text { FAM98B } \\ \text { CLIP4 } & \text { FBN1 } \\ \text { CLOCK } & \text { FBRS } \\ \text { CLU } & \text { FEM1B } \\ \text { CNTRL } & \\ & \end{array}$




$\begin{array}{cc}\text { ATP2A2 } & \text { COBLL1 } \\ \text { ATP6V0A1 } & \text { COL4A1 } \\ \text { ATP6V1F } & \text { COL5A2 } \\ \text { ATP6V1G1 } & \text { CORO2A } \\ \text { ATXN1L } & \text { COX20 } \\ \text { ATXN2 } & \text { CPEB3 } \\ \text { ATXN2L } & \text { CPM } \\ \text { ATXN7L3 } & \text { CSNK1A1 } \\ \text { ATXN7L3B } & \text { CXCL10 } \\ \text { AUP1 } & \text { CYBRD1 } \\ \text { AURKA } & \text { CYCS } \\ \text { AURKB } & \text { CYP4V2 } \\ \text { BACH1 } & \text { DAAM1 } \\ \text { BAG5 } & \text { DAXX } \\ \text { BAG6 } & \text { DCAF10 } \\ \text { BAHD1 } & \text { DCAF8 } \\ \text { BAZ1A } & \text { DCP1A } \\ \text { BAZ1B } & \text { DDAH1 } \\ \text { BAZ2A } & \text { DDHD2 } \\ \text { BBS7 } & \text { DDR2 } \\ \text { BCAS4 } & \text { DDX3X } \\ \text { BCAT1 } & \text { DDX46 } \\ \text { BCL7A } & \text { DDX55 } \\ \text { BCOR } & \text { DERL1 } \\ \text { BCORL1 } & \text { DICER1 } \\ \text { BEND4 } & \text { DLG1 } \\ \text { BFSP1 } & \text { DMD } \\ \text { BGLAP } & \text { DMTF1 } \\ \text { BIRC5 } & \text { DNAJC10 } \\ \text { BIRC6 } & \text { DNAJC16 } \\ \text { BMP7 } & \text { DOCK10 } \\ \text { BNIP3L } & \text { DOCK4 } \\ \text { BRD2 } & \text { DOCK5 }\end{array}$

FGB

FGF19

FGG

FJX1

FOS

FOXN2

FOXN3

FOXO3

FRK

FSCN1

GANAB

GAREM

GAREML

GAS2L3

GATAD2B

GEM

GLDN

GLUL

GOLGA7

GPR180

GPR82

GPR85

GRHL1

GTPBP10

H6PD

HBP1

HDGF

HECW1

HINFP

HLF

HNRNPM

HOXA10

HRH1 


$\begin{array}{cc}\text { BRI3BP } & \text { DOCK7 } \\ \text { BRPF1 } & \text { DSE } \\ \text { BRPF3 } & \text { DTX3L } \\ \text { BTBD9 } & \text { DUSP10 } \\ \text { BTG1 } & \text { DUSP8 } \\ \text { BTG2 } & \text { DYNC1L12 } \\ \text { BZW1 } & \text { E2F1 } \\ \text { BZW2 } & \text { E2F2 } \\ \text { C10orf118 } & \text { E2F3 } \\ \text { C11orf52 } & \text { ECI2 } \\ \text { C11orf57 } & \text { EDIL3 } \\ \text { C12orf4 } & \text { EGFR } \\ \text { C12orf49 } & \text { EIF1AX } \\ \text { C19orf47 } & \text { EIF2S1 } \\ \text { C19orf53 } & \text { EIF4A2 } \\ \text { C1GALT1 } & \text { EIF4EBP2 } \\ \text { C1RL } & \text { EIF5 } \\ \text { C1orf21 } & \text { ELAVL4 } \\ \text { C1orf210 } & \text { ELMO1 } \\ \text { C1orf27 } & \text { ELOVL4 } \\ \text { C1orf52 } & \text { ELOVL7 } \\ \text { C2orf44 } & \text { ENAH } \\ \text { C5orf15 } & \text { EPHA4 } \\ \text { C5orf24 } & \text { EPM2A } \\ \text { C5orf51 } & \text { EPM2AIP1 } \\ \text { C6orf62 } & \text { ERBB2 } \\ \text { C9orf156 } & \text { ERP44 } \\ \text { CA12 } & \text { ESYT2 } \\ \text { CACNG8 } & \text { ETNK1 } \\ \text { CALCOCO2 } & \text { EXOC5 } \\ \text { CALU } & \text { EXOC8 } \\ \text { CAPG } & \text { FAM126B } \\ \text { CARHSP1 } & \text { FAM136A }\end{array}$

HSD17B12
HUWE1
IFRD1
IGF1
IKZF4
IMPDH1
INSIG1
ISG20L2
ITGA11
ITGA6
ITIH5
IYD
KCNN3
KCTD15
KLF4
KLHDC3
KREMEN2
LAMC2
LDOC1L
LEPRE1
LILRB2
LIMS1
LOX
LPL
MAPK6
MAPKBP1
MARCH6
MAZ
MCL1
MDM2
MMP2
MORF4L1
MORF4L2




CBFB
CBX5
CBX6
CCBL2
CCDC115
CCDC134
CCDC71
CCNA1
CCNA2
CCNB1
CCNB2
CCND1
CCND2
CCND3
CCNF
CCNG1
CCNJ
CCNK
CCNT2
CCNY
CD151
CD2BP2
CD59
CD81
CD99
CDC25A
CDC34
CDCA7
CDCA8
CDIPT
CDK6
CDKAL1
CDKN1A

FAM20B
FAM217B
FAM3C
FAM46A
FANCl
FAS
FASLG
FAXDC2
FBXL13
FBXL17
FBX18
FBXL2
FBXO11
FBXO3
FERMT2
FGF12
FGFRL1
FIGN
FILIP1L
FKBP5
FMOD
FMR1
FNBP1
FOXN2
FOXN3
FOXO3
FRS2
FUBP1
FUT2
GAPVD1
GAS1
GDF5
GDPGP1

MTPN

MXD1

MYCN

N4BP2L2

NAA40

NASP

NAV3

NDUFB5

NDUFV3

NEDD9

NFIA

NKIRAS2

NRIP1

NTMT1

NUP93

NWD1

OAZ1

OTUD4

PAN2

PAQR3

PDGFRB

PER1

PHACTR2

PIGS

PIK3R1

PLAC8

PLAG1

PMAIP1

POLD3

POLL

PPM1D

PPP1R12B

PPP1R13B 


CDKN1B
CDV3
CELF1
CENPB
CENPV
CEP120
CEP135
CHAF1A
CHD1
CHD3
CHD4
CHD7
CHMP2A
CHMP3
CHPF2
CHRAC1
CHTOP
CIAO1
CIZ1
CKAP2
CKB
CKS2
CLCA2
CLDN12
CLINT1
CLPX
CLUH
CMC1
CNDP2
CNNM3
CNOT1
CNOT2
COIL

GGCX
GID4
GK5
GLCCI1
GLG1
GNAQ
GNB4
GNE
GOLGA4
GP5
GPAM
GPD1L
GPD2
GPR137C
GPR64
GRPEL2
GTF2A1
GTF2I
GXYLT2
HAPLN1
HECTD1
HERPUD2
HIC2
HIF1A
HIPK3
HIVEP3
HMGB3
HNRNPH1
HNRNPK
HOXA9
HPGD
HPS5
HS3ST3B1

PPP3CB
PPT1
PPY
PROSER2
PRY
PRY2
PTEN
PTP4A1
PTPLAD1
PTPRT
PUM1
PXDN
R3HDM4
RAB11FIP1
RAB30
RAB40C
RAET1L
RALGAPB
RAN
RBM20
RCC2
REL
REST
RET
RIOK3
RNASEL
RNF138
RNMTL1
RPF2
RPL22
RPS4X
RPS6KA3




$\begin{array}{cc}\text { COL3A1 } & \text { ICAM1 } \\ \text { COL8A1 } & \text { IGF1R } \\ \text { COLEC12 } & \text { IL12A } \\ \text { COMMD9 } & \text { IL1B } \\ \text { COPB2 } & \text { IPP } \\ \text { COPG1 } & \text { IRAK1 } \\ \text { COPS2 } & \text { IREB2 } \\ \text { COX6B1 } & \text { IRS4 } \\ \text { COX7B } & \text { ISCU } \\ \text { CPA4 } & \text { ITGB8 } \\ \text { CPEB1 } & \text { ITK } \\ \text { CPEB3 } & \text { ITSN2 } \\ \text { CPEB4 } & \text { IVNS1ABP } \\ \text { CPED1 } & \text { JAG1 } \\ \text { CPSF1 } & \text { JMY } \\ \text { CPSF3L } & \text { JPH1 } \\ \text { CREB1 } & \text { KAT6A } \\ \text { CREBRF } & \text { KBTBD6 } \\ \text { CREBZF } & \text { KBTBD7 } \\ \text { CRKL } & \text { KIAA1551 } \\ \text { CRY2 } & \text { KIAA1715 } \\ \text { CS } & \text { KIF6 } \\ \text { CSNK1D } & \text { KIFAP3 } \\ \text { CSNK2A1 } & \text { KLF5 } \\ \text { CSTF2 } & \text { KLF9 } \\ \text { CTBP2 } & \text { KLHL15 } \\ \text { CTCF } & \text { KLHL24 } \\ \text { CTHRC1 } & \text { KLHL42 } \\ \text { CTPS1 } & \text { KLK2 } \\ \text { CTR9 } & \text { KRIT1 } \\ \text { CUL1 } & \text { LAMP2 } \\ \text { CUL2 } & \text { LARS } \\ \text { CUL3 } & \text { LATS1 }\end{array}$

RRAGC

S100B

SAPCD2

SARS

SEC31A

SERPINB9

SERPINH1

SFRP2

SGK1

SH3GLB1

SLC16A1

SLC1A5

SLC24A2

SLC25A16

SLC29A2

SLC2A14

SLC38A7

SNX24

SOWAHB

SP2

SPARC

SPOPL

SPRTN

SRGAP2

SRPX2

STK17A

SUV420H2

SYNCRIP

TDG

TECPR2

TESPA1

TET1

TET2 


CUX1
CYP1A2
CYP2J2
CYSTM1
DAB2IP
DBNDD2
DBNL
DCAF7
DCD
DCTD
DCTPP1
DDX10
DDX17
DDX18
DDX20
DDX21
DDX28
DDX41
DDX49
DEDD
DENND4B
DENR
DFFA
DHTKD1
DHX16
DHX33
DHX57
DHX9
DIABLO
DIAPH1
DICER1
DIP2A
DISC1

LCLAT1

TET3

LCORL

LIFR

TGFB3

LIMCH1

LIN7C

LMBR1

LONRF2

LPGAT1

LRRC57

LRRFIP1

LYRM7

MALT1

MAP2K3

MAP3K1

MAP3K2

MARCKS

MAT2A

MAT2B

MBNL1

MCMDC2

MDM4

MED25

MED9

MEF2A

MEF2C

MEGF9

MEIS1

MGA

MGAT4A

MIB1

MKNK2

MMP2

MMP9

TMEM237

TMOD3

TMPRSS15

TMSB4X

TMTC3

TNFAIP3

TOR1A

TRAF4

TRAM2

TRIM63

TRIM68

TRIM72

TUBB2A

UBE2D4

ULBP2

VAMP3

VDAC1

VEGFA

VHL

WBSCR16

WDR26

WNK3

WWTR1

XIAP

YAE1D1

YY2

ZBTB20

ZBTB5

ZCCHC24

ZFP36

ZFP91 


$\begin{array}{cc}\text { DLAT } & \text { MOAP1 } \\ \text { DLC1 } & \text { MON2 } \\ \text { DLX2 } & \text { MORC3 } \\ \text { DMD } & \text { MOXD1 } \\ \text { DNA2 } & \text { MPP5 } \\ \text { DNAH9 } & \text { MRAP2 } \\ \text { DNAJC1 } & \text { MRPS10 } \\ \text { DNAJC11 } & \text { MSH2 } \\ \text { DNAJC28 } & \text { MSH6 } \\ \text { DNAJC8 } & \text { MTAP } \\ \text { DNAL1 } & \text { MTMR12 } \\ \text { DNM3 } & \text { MTMR9 } \\ \text { DNMBP } & \text { MTPN } \\ \text { DOCK5 } & \text { MUC1 } \\ \text { DPF2 } & \text { MYC } \\ \text { DPYS5 } & \text { MYCBP2 } \\ \text { DRAXIN } & \text { MYD88 } \\ \text { DRG2 } & \text { MYEF2 } \\ \text { DSG2 } & \text { MYO9A } \\ \text { DSP } & \text { NAA30 } \\ \text { DTX3L } & \text { NAA50 } \\ \text { DUSP1 } & \text { NBEA } \\ \text { DUSP12 } & \text { NCAM2 } \\ \text { DUSP23 } & \text { NCAPG } \\ \text { DUSP6 } & \text { NCOA3 } \\ \text { DVL3 } & \text { NCSTN } \\ \text { DYNC1H1 } & \text { NEK1 } \\ \text { DZIP1 } & \text { NETO2 } \\ \text { E2F3 } & \text { NFAT5 } \\ \text { E2F5 } & \text { NFIA } \\ \text { E2F6 } & \text { NFIB } \\ \text { E2F7 } & \text { NFKB1 } \\ \text { E2F8 } & \end{array}$

MTMR9

MYC

YYCBP2

MYEF2

MYO9A

NBEA

NCOA3

ETO2

NFIB

NIN 


$\begin{array}{cc}\text { EAF1 } & \text { NIPBL } \\ \text { ECHDC1 } & \text { NKTR } \\ \text { EDEM3 } & \text { NOC3L } \\ \text { EDN1 } & \text { NR2C2 } \\ \text { EEF1A1 } & \text { NSUN2 } \\ \text { EEF1E1 } & \text { NT5C2 } \\ \text { EEF2 } & \text { NTF3 } \\ \text { EFCAB14 } & \text { NUBPL } \\ \text { EFHD2 } & \text { NUFIP2 } \\ \text { EHD4 } & \text { NUP98 } \\ \text { EIF2AK1 } & \text { OLR1 } \\ \text { EIF2B3 } & \text { ORC4 } \\ \text { EIF3C } & \text { OSBPL1A } \\ \text { EIF3D } & \text { OSBPL3 } \\ \text { EIF4A1 } & \text { OSR1 } \\ \text { EIF4A2 } & \text { OTUD1 } \\ \text { EIF4A3 } & \text { PAG1 } \\ \text { EIF4G2 } & \text { PALLD } \\ \text { ELK4 } & \text { PAN3 } \\ \text { ELMOD2 } & \text { PARP1 } \\ \text { ELOVL1 } & \text { PARP9 } \\ \text { EMC6 } & \text { PATE2 } \\ \text { EMILIN2 } & \text { PBRM1 } \\ \text { ENG } & \text { PBX1 } \\ \text { ENTPD4 } & \text { PCBP1 } \\ \text { ENTPD6 } & \text { PDCD4 } \\ \text { EP300 } & \text { PDGFD } \\ \text { EPB41L3 } & \text { PDHA2 } \\ \text { EPHA4 } & \text { PELI1 } \\ \text { ERAP2 } & \text { PER2 } \\ \text { ERC1 } & \text { PGRB } \\ \text { ERCC1 } & \\ \text { ERGIC2 } & \\ & \end{array}$




\begin{tabular}{cc} 
ERGIC3 & PHACTR2 \\
ERO1L & PHF16 \\
ESPL1 & PHF17 \\
ETFA & PHF20 \\
F2 & PHF20L1 \\
FADS2 & PHIP \\
FAM104A & PHTF1 \\
FAM105A & PIAS3 \\
FAM126A & PIGN \\
FAM131A & PIGX \\
FAM136A & PIK3C2A \\
FAM178A & PIK3R1 \\
FAM203A & PITHD1 \\
FAM213A & PKD2 \\
FAM222B & PKNOX1 \\
FAM43A & PLAT \\
FAM49B & PLD1 \\
FAM57A & PLEKHA1 \\
FAM83G & PLEKHA2 \\
FAM84B & PLEKHA8 \\
FAM96A & PLOD3 \\
FANCD2 & PM20D2 \\
FARP1 & POLR3B \\
FBXL20 & PPAP2A \\
FBXW2 & PPARA \\
FEN1 & PPFIA4 \\
FGFRL1 & PPIF \\
FIGN & PPM1L \\
FLAD1 & PREPL \\
FLII & PRICKLE2 \\
FLNA & PRIM1 \\
FMNL3 & PRKAB2 \\
FMO4 & PRKCE \\
& \\
\hline
\end{tabular}




$\begin{array}{cc}\text { FNDC3A } & \text { PROSER1 } \\ \text { FOXK1 } & \text { PRPF39 } \\ \text { FOXRED1 } & \text { PRRC1 } \\ \text { FOXRED2 } & \text { PTAR1 } \\ \text { FPR1 } & \text { PTBP3 } \\ \text { FSTL1 } & \text { PTEN } \\ \text { FTO } & \text { PTGFR } \\ \text { FUT10 } & \text { PTK2 } \\ \text { FXN } & \text { PTPDC1 } \\ \text { FXR2 } & \text { PTPN14 } \\ \text { FZD5 } & \text { PTPN3 } \\ \text { FZD9 } & \text { PTX3 } \\ \text { GABPB1 } & \text { PURA } \\ \text { GABPB2 } & \text { PURB } \\ \text { GABRG1 } & \text { PURG } \\ \text { GALNT11 } & \text { RAB11FIP2 } \\ \text { GALNT2 } & \text { RAB22A } \\ \text { GAPDH } & \text { RAB32 } \\ \text { GAPVD1 } & \text { RAB6A } \\ \text { GATA6 } & \text { RAB6C } \\ \text { GATC } & \text { RABGAP1 } \\ \text { GBF1 } & \text { RAI14 } \\ \text { GCN1L1 } & \text { RALGPS2 } \\ \text { GDE1 } & \text { RAPGEF6 } \\ \text { GDF11 } & \text { RAPH1 } \\ \text { GDPD5 } & \text { RASA1 } \\ \text { GEMIN5 } & \text { RASEF } \\ \text { GEMIN7 } & \text { RASGRP1 } \\ \text { GFM2 } & \text { RASGRP3 } \\ \text { GGA3 } & \text { RB1 } \\ \text { GGCT } & \text { RDH11 } \\ \text { GGPS1 } & \text { RECK } \\ \text { GLB1 } & \text { REST }\end{array}$




$\begin{array}{cc}\text { GLO1 } & \text { REV1 } \\ \text { GLTPD1 } & \text { REV3L } \\ \text { GLTSCR2 } & \text { RFFL } \\ \text { GNAS } & \text { RHO } \\ \text { GNB1 } & \text { RHOB } \\ \text { GNG5 } & \text { RHOQ } \\ \text { GOLGA4 } & \text { RMND5A } \\ \text { GPATCH1 } & \text { RNF103 } \\ \text { GPATCH4 } & \text { RNF11 } \\ \text { GPHN } & \text { RNF111 } \\ \text { GPI } & \text { RNF149 } \\ \text { GPM6B } & \text { RNF185 } \\ \text { GPR56 } & \text { RNF6 } \\ \text { GPRIN3 } & \text { RP2 } \\ \text { GPX7 } & \text { RPRD2 } \\ \text { GREM1 } & \text { RPS6KA3 } \\ \text { GRPEL2 } & \text { RPS7 } \\ \text { GSG2 } & \text { RRAGC } \\ \text { GSK3A } & \text { RSF1 } \\ \text { GSK3B } & \text { RSPRY1 } \\ \text { GSPT1 } & \text { RTN4 } \\ \text { GSR } & \text { RUFY3 } \\ \text { GTF21 } & \text { SACM1L } \\ \text { GTF3C1 } & \text { SALL1 } \\ \text { GTF3C4 } & \text { SAMD5 } \\ \text { GTPBP3 } & \text { SAR1A } \\ \text { GYG1 } & \text { SASH1 } \\ \text { GYS1 } & \text { SATB1 } \\ \text { GYS2 } & \text { SCAF11 } \\ \text { GZF1 } & \text { SCRN1 } \\ \text { HADHA } & \text { SEC63 } \\ \text { HAND1 } & \text { SECISBP2L } \\ \text { HARS } & \text { SEMA5A }\end{array}$




$\begin{array}{cc}\text { HAT1 } & \text { SEPT2 } \\ \text { HAUS6 } & \text { SERAC1 } \\ \text { HBP1 } & \text { SERPINB5 } \\ \text { HCFC1 } & \text { SERPINI1 } \\ \text { HEATR2 } & \text { SESN1 } \\ \text { HELLS } & \text { SESTD1 } \\ \text { HERPUD1 } & \text { SET } \\ \text { HES1 } & \text { SETD1B } \\ \text { HGS } & \text { SETD2 } \\ \text { HIF1A } & \text { SF1 } \\ \text { HIPK1 } & \text { SFXN1 } \\ \text { HIST1H1C } & \text { SGCB } \\ \text { HIST1H2BD } & \text { SGK1 } \\ \text { HIST1H2BK } & \text { SGK3 } \\ \text { HIST1H3B } & \text { SGTB } \\ \text { HK1 } & \text { SH3GLB1 } \\ \text { HMGA1 } & \text { SIRT2 } \\ \text { HMGA2 } & \text { SKP2 } \\ \text { HMGB1 } & \text { SLAIN2 } \\ \text { HMGB2 } & \text { SLC16A10 } \\ \text { HMGCS1 } & \text { SLC17A5 } \\ \text { HNRNPDL } & \text { SLC26A2 } \\ \text { HNRNPF } & \text { SLC31A1 } \\ \text { HNRNPL } & \text { SLC5A3 } \\ \text { HNRNPUL1 } & \text { SLC9A6 } \\ \text { HOXD11 } & \text { SLK } \\ \text { HRAS } & \text { SLMAP } \\ \text { HS2ST1 } & \text { SMAD1 } \\ \text { HSF2 } & \text { SMAD7 } \\ \text { HSP90AA1 } & \text { SMARCA4 } \\ \text { HSPA1B } & \text { SMC1A } \\ \text { HSPA8 } & \text { SMN1 } \\ \text { HTT } & \text { SMNDC1 }\end{array}$




HUWE1
IARS2
IBA57
ICOSLG
IDI1
IFIT5
IFNB1
IFNLR1
IFRD1
IGDCC4
IGF1R
IGF2BP1
IGF2BP2
IGF2BP3
IGHMBP2
IGSF3
IGSF8
IKZF3
IL17RC
IMPDH1
IMPDH2
INPPL1
INTS1
INTS5
INTS7
IP6K1
IPCEF1
IPO11
IPO4
IPO7
IPO8
IPO9
IRF2BP2

SNRK

SNRNP48

SNX13

SNX30

SOCS4

SOCS5

SOD3

SOWAHC

SOX11

SOX2

SOX4

SOX5

SP1

SPATS2L

SPG11

SPIN1

SPPL3

SPRY2

SPRY4

SPTLC3

SREK1

SREK1IP1

SRPK2

SRSF11

SSFA2

ST13

ST6GAL1

STAG2

STARD13

STAT3

STRBP

STXBP5

SUV420H1 


$\begin{array}{cc}\text { IRS2 } & \text { SUZ12 } \\ \text { IRS4 } & \text { SYNE2 } \\ \text { ISOC2 } & \text { TAF1 } \\ \text { ITGA3 } & \text { TAF5 } \\ \text { ITGB5 } & \text { TBL1XR1 } \\ \text { JAG1 } & \text { TCEANC2 } \\ \text { KANSL1L } & \text { TCF21 } \\ \text { KCNC4 } & \text { TESK2 } \\ \text { KCTD12 } & \text { TET1 } \\ \text { KCTD21 } & \text { TGFB1 } \\ \text { KDELR1 } & \text { TGFB2 } \\ \text { KDM4A } & \text { TGFBI } \\ \text { KHSRP } & \text { TGFBR2 } \\ \text { KIAA0141 } & \text { TGFBR3 } \\ \text { KIAA0391 } & \text { TGIF1 } \\ \text { KIAA0930 } & \text { THOC2 } \\ \text { KIAA1328 } & \text { TIAM1 } \\ \text { KIAA1377 } & \text { TIMP3 } \\ \text { KIAA1429 } & \text { TLR4 } \\ \text { KIAA1549 } & \text { TM9SF3 } \\ \text { KIAA1586 } & \text { TMEM147 } \\ \text { KIAA1715 } & \text { TMEM170A } \\ \text { KIAA2018 } & \text { TMEM2 } \\ \text { KIDINS220 } & \text { TMEM241 } \\ \text { KIF1C } & \text { TMEM245 } \\ \text { KIF27 } & \text { TMEM56 } \\ \text { KIF2A } & \text { TMX4 } \\ \text { KIFC1 } & \text { TNFAIP3 } \\ \text { KLF10 } & \text { TNFRSF10B } \\ \text { KLHDC8B } & \text { TNPO1 } \\ \text { KLHL11 } & \text { TNRC6B } \\ \text { KLHL15 } & \\ \text { KMT2D } & \\ & \\ & \end{array}$




\begin{tabular}{|c|c|}
\hline KPNA5 & TOP2A \\
\hline KPNA6 & TOPORS \\
\hline KREMEN1 & TOR1AIP2 \\
\hline KXD1 & TP53BP2 \\
\hline L3MBTL4 & TP63 \\
\hline LACE1 & TPM1 \\
\hline LACTB2 & TPRG1L \\
\hline LARP1 & TRAPPC2 \\
\hline LBR & TRIM14 \\
\hline LCOR & TRIM2 \\
\hline LDLR & TRIM33 \\
\hline LEFTY1 & TRIM38 \\
\hline LIMD2 & TRIM44 \\
\hline LIN28A & TRIM59 \\
\hline LIN28B & TRPM7 \\
\hline LLPH & TSHZ3 \\
\hline LMAN2 & TSNAX \\
\hline LPCAT3 & TTC33 \\
\hline LPGAT1 & TUBGCP5 \\
\hline LPHN2 & TWSG1 \\
\hline LPL & UBA1 \\
\hline LRIG1 & UBR3 \\
\hline LRIG3 & UBR5 \\
\hline LRRC20 & UGGT1 \\
\hline LRRC40 & UHRF1BP1 \\
\hline LRRC41 & UQCRB \\
\hline LRRC47 & USP34 \\
\hline LRRC8A & USP47 \\
\hline LSG1 & USP7 \\
\hline LSM6 & UTRN \\
\hline LTA4H & VASH2 \\
\hline LYN & VEGFA \\
\hline LZIC & VHL \\
\hline
\end{tabular}




$\begin{array}{cc}\text { MAB21L1 } & \text { VPS13A } \\ \text { MANSC1 } & \text { VPS26A } \\ \text { MAP2K2 } & \text { VPS36 } \\ \text { MAP2K7 } & \text { VPS54 } \\ \text { MAP4 } & \text { WDR7 } \\ \text { MAP7 } & \text { WFS1 } \\ \text { MAP7D1 } & \text { WHSC1 } \\ \text { MAPK1 } & \text { WHSC1L1 } \\ \text { MAPK6 } & \text { WIBG } \\ \text { MARCH6 } & \text { WNK1 } \\ \text { MARS } & \text { WNK3 } \\ \text { MARS2 } & \text { WNT5A } \\ \text { MAT2B } & \text { WSB1 } \\ \text { MBD2 } & \text { WWC2 } \\ \text { MBD6 } & \text { WWP1 } \\ \text { MBNL1 } & \text { YME1L1 } \\ \text { MCAT } & \text { YOD1 } \\ \text { MCF2L2 } & \text { ZADH2 } \\ \text { MCM4 } & \text { ZBTB20 } \\ \text { MCM7 } & \text { ZBTB38 } \\ \text { MDM2 } & \text { ZBTB46 } \\ \text { MDM4 } & \text { ZBTB47 } \\ \text { MED13 } & \text { ZBTB8A } \\ \text { MED14 } & \text { ZCCHC3 } \\ \text { MED25 } & \text { ZFYVE16 } \\ \text { MED28 } & \text { ZMYM2 } \\ \text { MEF2C } & \text { ZNF132 } \\ \text { MESDC1 } & \text { ZNF207 } \\ \text { MFSD3 } & \text { ZNF217 } \\ \text { MFSD8 } & \text { ZNF267 } \\ \text { MGAT4C } & \text { ZNF292 } \\ \text { MGME1 } & \text { ZNF326 } \\ \text { MIB1 } & \\ & \\ \text { MNF35 }\end{array}$


ZNF618

ZNF667

ZNF793

MLLT1

ZRANB1

MLLT10

ZYG11B

MMS22L

MOB1A

MOB1B

MOV10

MPG

MPND

MRM1

MRPL12

MRPL35

MRPL37

MRPL47

MRPS11

MRPS24

MRPS33

MSANTD2

MSH6

MSI1

MSI2

MSMO1

MSN

MTFP1

MTFR1

MTPN 
MTRR

MTSS1L

MTUS1

MTX3

MXD1

MYADM

MYC

MYCBP

MY01C

MYO1E

MYOM2

NAA10

NAA15

NAA20

NAA25

NAA30

NAA40

NAA50

NABP2

NACA

NAF1

NAP1L1

NARS2

NAT8L

NBPF15

NCAPD2

NCAPG2

NCKIPSD

NCOA3

NCOA5

NCOA7

NCOR1

NDRG1 
NDUFA10

NDUFAF7

NEDD4

NEK9

NFATC1

NFATC3

NFKBIA

NFU1

NHLRC2

NHLRC3

NHSL1

NIPBL

NLE1

NME4

NME6

NOA1

NOL6

NOLC1

NOM1

$\mathrm{NOMO} 3$

NOP14

NPTN

NR2E1

NR6A1

NRAS

NRBF2

NRD1

NSA2

NSD1

NSMCE4A

NSUN2

NT5DC2

NUBP2 


$$
\begin{aligned}
& \text { NUCB2 } \\
& \text { NUCKS1 } \\
& \text { NUDT19 } \\
& \text { NUP153 } \\
& \text { NUP155 } \\
& \text { NUP214 } \\
& \text { NUP35 } \\
& \text { NUSAP1 } \\
& \text { NVL } \\
& \text { NXN } \\
& \text { NXT2 } \\
& \text { OCIAD1 } \\
& \text { OCLN } \\
& \text { ONECUT2 } \\
& \text { OPA3 } \\
& \text { OPRL1 } \\
& \text { ORC4 } \\
& \text { OSBPL10 } \\
& \text { OSTM1 } \\
& \text { OTUB1 } \\
& \text { OXA1L } \\
& \text { OXNAD1 } \\
& \text { OXR1 } \\
& \text { PA2G4 } \\
& \text { PAAF1 } \\
& \text { PABPC1 } \\
& \text { PAFAH1B3 } \\
& \text { PAFAH2 } \\
& \text { PAK1 } \\
& \text { PALD1 } \\
& \text { PAPOLA } \\
& \text { PAPOLG } \\
& \text { PAPSS1 }
\end{aligned}
$$


PARP16

PARVB

PAX3

PCBP2

PCCB

PCGF3

PCTP

PCYT1A

PCYT1B

PCYT2

PDCD10

PDCD11

PDCL3

PDE12

PDGFRA

PDHX

PDK1

PDP2

PDPR

PDS5A

PDZD8

PEG10

PER1

PEX11B

PEX6

PFKM

PFN1

PGGT1B

PGM1

PGM2L1

PGM3

PGRMC1

PHACTR4 
PHF8

PHKA1

PIGG

PIGU

PIH1D1

PITHD1

PLAGL2

PLCB3

PLCG2

PLEKHA3

PLEKHO1

PLK1

PLXDC2

PLXNB2

PLXND1

PM20D2

PMAIP1

PMF1

PMPCA

POLA2

POLD2

POLR1A

POLR1B

POLR2A

POLR2C

POLR2D

POLR2H

POLR2L

POLR3B

POLR3D

POLR3G

POM121

POM121C 
POP1

POTEG

PPARGC1A

PPID

PPIL3

PPIL4

PPM1G

PPP1R10

PPP1R12C

PPP1R15B

PPP1R16B

PPP1R7

PPP2R2A

PPP2R5E

PPRC1

PRAF2

PRDM1

PRDM4

PRIM1

PRIM2

PRKAA2

PRKAR2A

PRKD1

PRPF19

PRPF8

PRPS1

PRRC2A

PRRC2B

PRSS22

PRTG

PSD3

PSMD9

PSME3 
PSMG4

PTCD1

PTGES2

PTGFRN

PTGS2

PTP4A2

PTPN23

PTTG1

PUM1

PURA

PUS1

PVR

PXDN

PYCRL

QARS

QDPR

QKI

QRSL1

RAB10

RAB11FIP4

RAB19

RAB38

RAB3GAP2

RAB $40 C$

RABL2A

RABL2B

RAD18

RAI1

RALB

RALGAPB

RANBP6

RAP2C

RBBP6 
RBFOX1

RBFOX2

RBM12

RBM12B

RBM19

RBM22

RBM34

RBPJ

RCAN1

RDH10

RDX

REEP4

REPS1

RFC2

RFFL

RHD

$\mathrm{RHOB}$

RHOG

RIOK2

RIOK3

RNASE10

RNF11

RNF115

RNF144B

RNF40

RNF44

RNFT1

RNMT

RNMTL1

RORA

RPAP1

RPIA

RPL12 
RPL18

RPL18A

RPP38

RPS16

RPS24

RPS4X

RPSA

RRAD

RRBP1

RRM1

RRM2

RRP1B

RRP7A

RRP8

RS1

RTCA

RUFY2

RUVBL2

RWDD1

RXRB

SAFB

SALL2

SALL3

SAR1A

SC5D

SCAF4

SCAF8

SCAMP1

SCAMP3

SCD

SCML2

SCRN1

SCYL1 
SDAD1

SDR42E1

SEC11A

SEC16A

SEC23B

SEC24A

SEMA4C

SEMA4D

SEMG2

SENP1

SEPT4

SERBP1

SETD5

SF3B1

SFMBT1

SFT2D3

SH3GLB1

SH3PXD2A

SHOC2

SIGMAR1

SIKE1

SIPA1L2

SKI

SLC10A3

SLC10A7

SLC11A2

SLC12A7

SLC16A14

SLC16A9

SLC19A3

SLC1A4

SLC20A1

SLC25A1 


SLC25A12
SLC25A13
SLC25A19
SLC25A24
SLC25A32
SLC25A4
SLC27A2
SLC30A1
SLC30A7
SLC35A5
SLC35F1
SLC35F6
SLC38A1
SLC38A2
SLC38A5
SLC38A7
SLC5A6
SLC9A3R1
SMARCA1
SMARCA4
SMARCAD1
SMARCB1
SMARCC1
SMARCC2
SMARCD1
SMC1A
SMCR8
SMG1
SMG7
SNAP23
SNRPA
SNRPE
SNX12


SNX17

SOCS1

SOD2

SON

SOX13

SOX9

SP1

SP100

SPAG9

SPATA12

SPCS3

SPN

SPR

SPRYD4

SPRYD7

SPTBN2

SRCAP

SRI

SSR1

ST13

STAT2

STEAP3

STIM1

STIP1

STK38

STK4

STRN

SUMO1

SUMO2

SUOX

SUPT16H

SUPT2OH

SURF4 
SYF2

SYNE1

SYNE2

SYNGR2

SYNJ2BP

SYT4

SZRD1

TAB2

TAF9B

TBC1D13

TBC1D15

TBC1D19

TBC1D20

TBRG4

TCOF1

TENM3

TERF2IP

TES

TGFBR1

TGFBR3

TGOLN2

THBS1

THEM6

THOC5

THYN1

TIAM1

TIMM23

TIMM50

TIMM8A

TIMM9

TJP1

TLN1

TLR4 

TM4SF1
TMED5
TMEM115
TMEM167A
TMEM2
TMEM201
TMEM33
TMEM55B
TMEM63B
TMEM65
TMTC3
TNFRSF10B
TNFSF12
TNFSF9
TNPO1
TNRC6B
TOE1
TOR1AIP2
TOR4A
TOX3
TP53INP1
TPBG
TPD52L2
TPM4
TPP2
TPT1
TRABD
TRAPPC1
TRIM24
TRIM28
TRIM71
TRIP12
TRMT1 
TRNT1

TROVE2

TRUB2

TSC22D2

TSEN15

TSPAN3

TST

TTC33

TTC9C

TTLL12

TUBA1B

TUBA1C

TUBB

TUBB2A

TUBGCP2

TUBGCP3

TUT1

TXLNA

TXLNG

TXNL4A

TYMS

UBA1

UBA3

UBAP2L

UBE2A

UBE2D2

UBE2D3

UBE2I

UBE2M

UBE2Q1

UBXN2B

UBXN8

UCK1 
UCK2

UGT8

UHRF1

UNC13B

UNC13D

USO1

USP10

USP14

USP15

USP22

USP38

USP54

UTP15

UTP6

UTRN

VAMP3

VCL

VEZF1

VPS28

VPS39

VPS41

VPS51

VWA8

VWA9

WARS2

WASF1

WASL

WBP11

WBSCR16

WDR25

WDR26

WDR3

WDR33 
WDR4

WDR55

WDR74

WDR75

WEE1

WLS

WNK1

XPO5

XPO7

XRN1

XYLT2

YAE1D1

YAP1

YEATS2

YIPF3

YLPM1

YOD1

YTHDC1

YWHAE

YWHAZ

ZADH2

ZBTB37

ZBTB5

ZBTB80S

ZC3H11A

ZC3HAV1

ZC3HAV1L

ZCCHC11

ZCCHC3

ZCCHC9

ZER1

ZFAND4

ZFHX4 
ZMYM2

ZNF106

ZNF136

ZNF148

ZNF181

ZNF200

ZNF207

ZNF256

ZNF264

ZNF28

ZNF3

ZNF317

ZNF417

ZNF426

ZNF443

ZNF460

ZNF507

ZNF546

ZNF556

ZNF566

ZNF578

ZNF581

ZNF584

ZNF585A

ZNF587

ZNF606

ZNF611

ZNF629

ZNF644

ZNF652

ZNF687

ZNF774

ZNF799 
ZNF8

ZNF800

ZNF805

ZNF841 


\section{ENTREZ ID GENE NAME}

10038

poly (ADP-ribose) polymerase 2

DnaJ (Hsp40) homolog, subfamily B, member

$10060 \quad$ ATP-binding cassette, sub-family C (CFTR/MRP), member 9

10165 solute carrier family 25 , member 13 (citrin)

$10228 \quad \underline{\operatorname{syntaxin} 6}$

$10241 \quad$ calcium binding and coiled-coil domain 2

$1027 \quad$ cyclin-dependent kinase inhibitor 1B (p27, Kip1)

$10342 \quad$ TRK-fused gene

$10521 \quad$ DEAD (Asp-Glu-Ala-Asp) box polypeptide 17

$10787 \quad$ NCK-associated protein

$10794 \quad$ zinc finger protein 460

10807 serologically defined colon cancer antigen 3; similar to Serologically defined colon cancer antigen 3

10818 fibroblast growth factor receptor substrate 2

$10892 \quad$ mucosa associated lymphoid tissue lymphoma translocation gene 1

10905 mannosidase, alpha, class $1 \mathrm{~A}$, member 2

10933 mortality factor 4; mortality factor 4 like 1

$11044 \quad$ polymerase (DNA directed) sigma

11080 DnaJ (Hsp40) homolog, subfamily B, member 4

$11136 \quad$ solute carrier family 7 (cationic amino acid transporter, $y+$ system), member 9

$11143 \quad$ MYST histone acetyltransferase 2: similar to MYST histone acetyltransferase 2

$11153 \quad$ FIC domain containing

11201 polymerase (DNA directed) iota

11238 inactivation escape 2 (non-protein coding); carbonic anhydrase VB, mitochondrial 


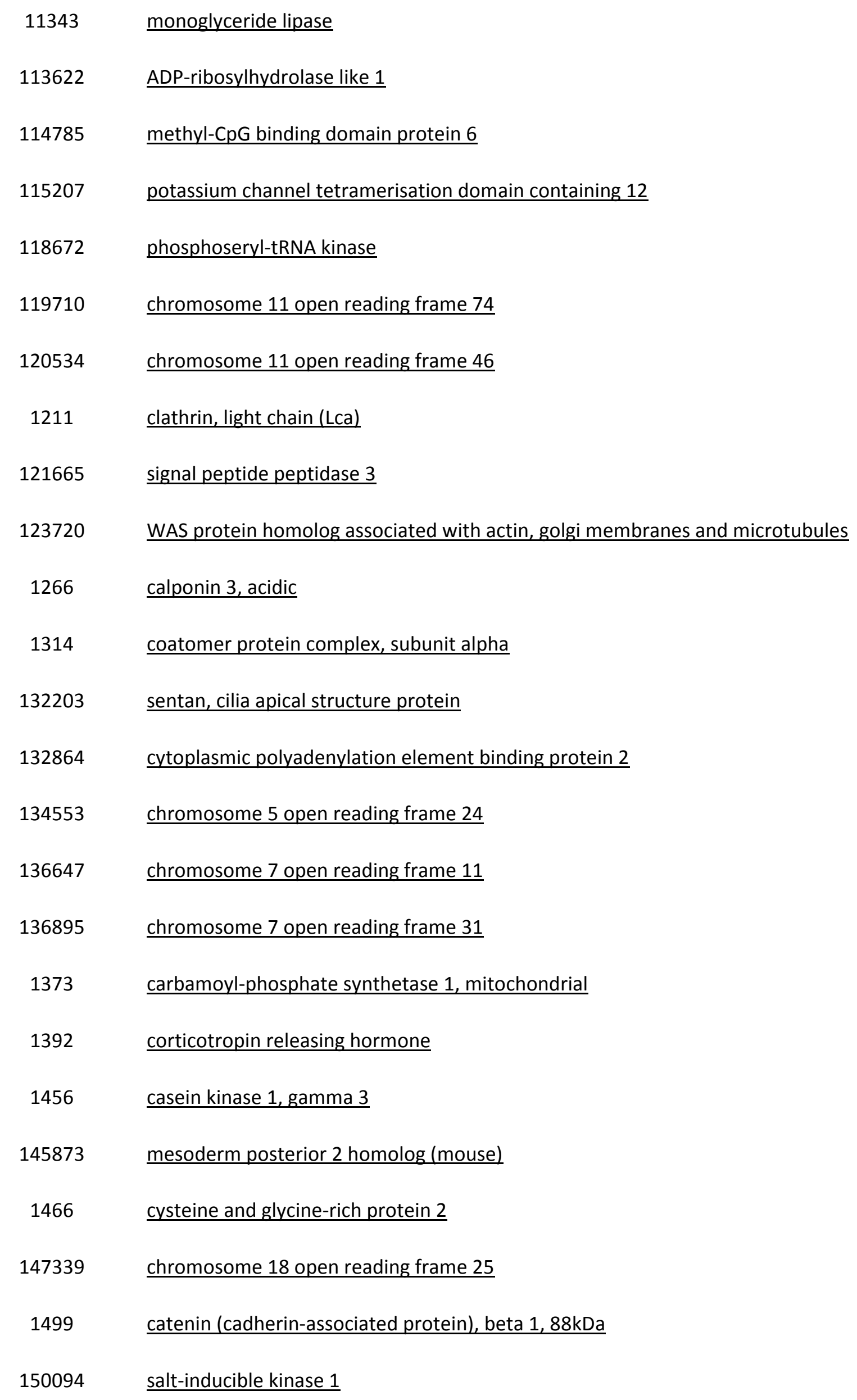




\begin{tabular}{|c|c|}
\hline 151188 & ADP-ribosylation-like factor 6 interacting protein 6 \\
\hline 152006 & ring finger protein 38 \\
\hline 159119 & heat shock transcription factor, $Y$-linked 1 ; heat shock transcription factor, $Y$ linked 2 \\
\hline 160418 & transmembrane and tetratricopeptide repeat containing 3 \\
\hline 1605 & dystroglycan 1 (dystrophin-associated glycoprotein 1) \\
\hline 161882 & zinc finger protein, multitype 1 \\
\hline 163223 & zinc finger protein 676 \\
\hline 163590 & torsin A interacting protein 2 \\
\hline 186 & angiotensin II receptor, type 2 \\
\hline 1894 & epithelial cell transforming sequence 2 oncogene \\
\hline 1959 & early growth response 2 \\
\hline 1983 & eukaryotic translation initiation factor 5 \\
\hline 200232 & chromosome 20 open reading frame 106 \\
\hline 200424 & tet oncogene family member 3 \\
\hline 203228 & chromosome 9 open reading frame 72 \\
\hline 205 & adenylate kinase 3-like 2; adenylate kinase 3-like 1 \\
\hline 2054 & $\underline{\text { syntaxin } 2}$ \\
\hline 2063 & nuclear receptor subfamily 2 , group $F$, member 6 \\
\hline 2099 & estrogen receptor 1 \\
\hline 210 & aminolevulinate, delta-, dehydratase \\
\hline 2124 & ecotropic viral integration site $2 \mathrm{~B}$ \\
\hline 2180 & acyl-CoA synthetase long-chain family member 1 \\
\hline 2182 & acyl-CoA synthetase long-chain family member 4 \\
\hline 219902 & transmembrane protein 136 \\
\hline 221079 & ADP-ribosylation factor-like $5 \mathrm{~B}$ \\
\hline
\end{tabular}




\begin{tabular}{|c|c|}
\hline 222068 & $\underline{\text { transmembrane emp24 protein transport domain containing } 4}$ \\
\hline 2239 & glypican 4 \\
\hline 22809 & activating transcription factor 5 \\
\hline 22820 & coatomer protein complex, subunit gamma \\
\hline 22862 & fibronectin type III domain containing $3 \mathrm{~A}$ \\
\hline 22876 & inositol polyphosphate-5-phosphatase F \\
\hline 22879 & MON1 homolog B (yeast) \\
\hline 22936 & elongation factor, RNA polymerase $I I, 2$ \\
\hline 23049 & SMG1 homolog, phosphatidylinositol 3-kinase-related kinase (C. elegans) \\
\hline 23059 & clusterin associated protein 1 \\
\hline 23118 & mitogen-activated protein kinase kinase kinase 7 interacting protein 2 \\
\hline 23171 & glycerol-3-phosphate dehydrogenase 1-like \\
\hline 23174 & zinc finger, $\mathrm{CCHC}$ domain containing 14 \\
\hline 23181 & DIP2 disco-interacting protein 2 homolog A (Drosophila) \\
\hline 23198 & proteasome (prosome, macropain) activator subunit 4 \\
\hline 23235 & salt-inducible kinase 2 \\
\hline 23350 & $\underline{\text { U2-associated SR140 protein }}$ \\
\hline 23531 & monocyte to macrophage differentiation-associated \\
\hline 23613 & zinc finger, MYND-type containing 8 \\
\hline 23657 & solute carrier family 7, (cationic amino acid transporter, $y+$ system) member 11 \\
\hline 23731 & chromosome 9 open reading frame 5 \\
\hline 23787 & mitochondrial carrier homolog 1 (C. elegans) \\
\hline 253260 & RPTOR independent companion of MTOR, complex 2 \\
\hline 253430 & inositol polyphosphate multikinase \\
\hline 254552 & nudix (nucleoside diphosphate linked moiety X)-type motif 8 \\
\hline
\end{tabular}




\begin{tabular}{|c|c|}
\hline 256471 & major facilitator superfamily domain containing 8 \\
\hline 25776 & chibby homolog 1 (Drosophila) \\
\hline 25823 & tryptase gamma 1 \\
\hline 25831 & $\underline{\mathrm{HECT}}$ domain containing 1 \\
\hline 25853 & $\underline{\text { WD repeat domain } 40 \mathrm{~A}}$ \\
\hline 25888 & $\underline{\text { zinc finger protein } 473}$ \\
\hline 25896 & integrator complex subunit 7 \\
\hline 259230 & sphingomyelin synthase 1 \\
\hline 259249 & MAS-related GPR, member X1 \\
\hline 25930 & protein tyrosine phosphatase, non-receptor type 23 \\
\hline 25932 & $\underline{\text { chloride intracellular channel } 4}$ \\
\hline 25940 & family with sequence similarity 98 , member $A$ \\
\hline 25948 & kelch repeat and BTB (POZ) domain containing 2 \\
\hline 25976 & $\underline{\text { TCDD-inducible poly(ADP-ribose) polymerase }}$ \\
\hline 26035 & glucuronic acid epimerase \\
\hline 26071 & family with sequence similarity 127 , member $B$ \\
\hline 26145 & interferon regulatory factor 2 binding protein 1 \\
\hline 2615 & leucine rich repeat containing 32 \\
\hline 26207 & phosphatidylinositol transfer protein, cytoplasmic 1 \\
\hline 26273 & F-box protein 3 \\
\hline 26503 & solute carrier family 17 (anion/sugar transporter), member 5 \\
\hline 2672 & growth factor independent 1 transcription repressor \\
\hline 27125 & AF4/FMR2 family, member 4 \\
\hline 27314 & $\underline{\text { RAB30, member RAS oncogene family }}$ \\
\hline 27436 & $\underline{\text { echinoderm microtubule associated protein like } 4}$ \\
\hline
\end{tabular}




\begin{tabular}{|c|c|}
\hline 2776 & guanine nucleotide binding protein ( $G$ protein), q polypeptide \\
\hline 2778 & GNAS complex locus \\
\hline 2783 & guanine nucleotide binding protein ( $G$ protein), beta polypeptide 2 \\
\hline 2800 & golgi autoantigen, golgin subfamily $a, 1$ \\
\hline 283635 & family with sequence similarity 177 , member A1 \\
\hline 285598 & ADP-ribosylation factor-like 10 \\
\hline 286148 & dpy-19-like 4 (C. elegans) \\
\hline 28982 & feline leukemia virus subgroup C cellular receptor 1 \\
\hline 29097 & cornichon homolog 4 (Drosophila) \\
\hline 29904 & eukaryotic elongation factor-2 kinase \\
\hline 29966 & $\underline{\text { striatin, calmodulin binding protein } 3}$ \\
\hline 31 & acetyl-Coenzyme A carboxylase alpha \\
\hline 3146 & high-mobility group box 1; high-mobility group box 1 -like 10 \\
\hline 3159 & hypothetical LOC100130009; high mobility group AT-hook 1 \\
\hline 3204 & homeobox A7 \\
\hline 3205 & $\underline{\text { homeobox A9 }}$ \\
\hline 3206 & homeobox A10 \\
\hline 3209 & $\underline{\text { homeobox A13 }}$ \\
\hline 324 & adenomatous polyposis coli \\
\hline 3281 & heat shock factor binding protein 1 \\
\hline 3295 & hydroxysteroid (17-beta) dehydrogenase 4 \\
\hline 3304 & heat shock $70 \mathrm{kDa}$ protein $1 \mathrm{~A}$; heat shock $70 \mathrm{kDa}$ protein $1 \mathrm{~B}$ \\
\hline 331 & $\underline{X \text {-linked inhibitor of apoptosis }}$ \\
\hline 338339 & C-type lectin domain family 4 , member $\mathrm{D}$ \\
\hline 339500 & zinc finger protein 678 \\
\hline
\end{tabular}




\begin{tabular}{|c|c|}
\hline 340547 & $\underline{\text { V-set and immunoglobulin domain containing } 1}$ \\
\hline 3455 & interferon (alpha, beta and omega) receptor 2 \\
\hline 3491 & cysteine-rich, angiogenic inducer, 61 \\
\hline 353500 & $\underline{\text { bone morphogenetic protein } 8 \mathrm{a}}$ \\
\hline 3552 & interleukin 1, alpha \\
\hline 3569 & interleukin 6 (interferon, beta 2) \\
\hline 3572 & interleukin 6 signal transducer (gp130, oncostatin M receptor) \\
\hline 3619 & inner centromere protein antigens $135 / 155 \mathrm{kDa}$ \\
\hline 3632 & inositol polyphosphate-5-phosphatase, $40 \mathrm{kDa}$ \\
\hline 3654 & interleukin-1 receptor-associated kinase 1 \\
\hline 3659 & interferon regulatory factor 1 \\
\hline 3685 & integrin, alpha V (vitronectin receptor, alpha polypeptide, antigen CD51) \\
\hline 374977 & chromosome 1 open reading frame 175 \\
\hline 3752 & potassium voltage-gated channel, Shal-related subfamily, member 3 \\
\hline 378 & ADP-ribosylation factor 4 \\
\hline 3798 & kinesin family member $5 \mathrm{~A}$ \\
\hline 3799 & kinesin family member $5 \mathrm{~B}$ \\
\hline 3842 & $\underline{\operatorname{transp} p o r t i n} 1$ \\
\hline 3915 & laminin, gamma 1 (formerly LAMB2) \\
\hline 4015 & $\underline{\text { lysyl oxidase }}$ \\
\hline 402055 & $\underline{\text { SRR1 domain containing }}$ \\
\hline 4026 & $\underline{\text { LIM domain containing preferred translocation partner in lipoma }}$ \\
\hline 406 & aryl hydrocarbon receptor nuclear translocator-like \\
\hline 4068 & $\underline{\mathrm{SH} 2 \text { domain protein } 1 \mathrm{~A}}$ \\
\hline 4082 & yristoylated alanine-rich protein kinase $\mathrm{C}$ substrate \\
\hline
\end{tabular}




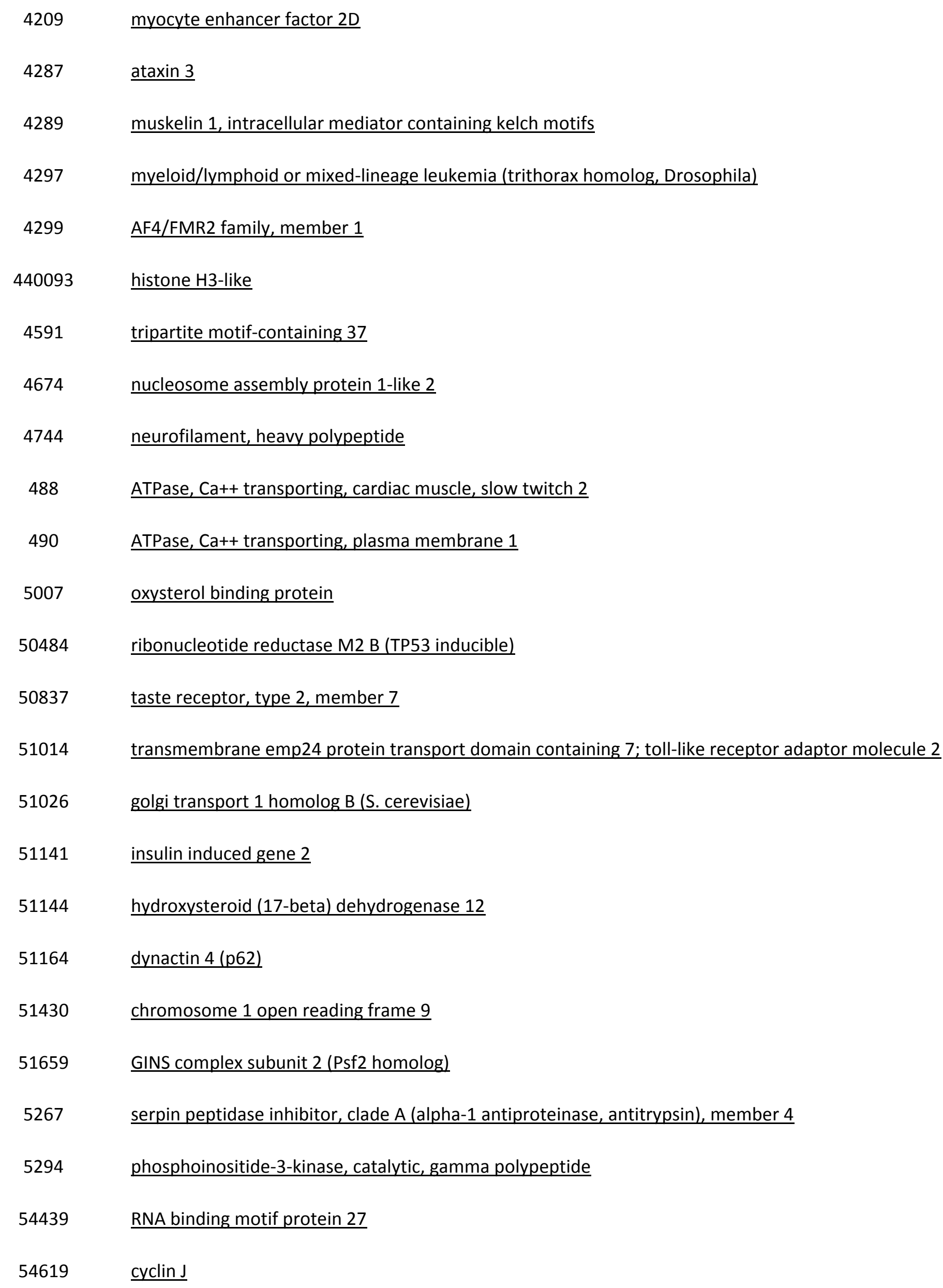




\begin{tabular}{|c|c|}
\hline 54622 & ADP-ribosylation factor-like 15 \\
\hline 54662 & $\underline{\text { TBC1 domain family, member } 13}$ \\
\hline 54726 & OTU domain containing 4 \\
\hline 54734 & RAB39, member RAS oncogene family \\
\hline 54906 & chromosome 10 open reading frame 18 \\
\hline 54978 & chromosome 2 open reading frame 18 \\
\hline 55038 & cell division cycle associated 4 \\
\hline 5504 & protein phosphatase 1, regulatory (inhibitor) subunit 2 \\
\hline 55054 & ATG16 autophagy related 16-like 1 (S. cerevisiae) \\
\hline 55119 & PRP38 pre-mRNA processing factor 38 (yeast) domain containing $B$ \\
\hline 55122 & $\underline{\text { akirin } 2}$ \\
\hline 55125 & centrosomal protein $192 \mathrm{kDa}$ \\
\hline 5522 & protein phosphatase 2 (formerly $2 A$ ), regulatory subunit $B$, gamma isoform \\
\hline 55224 & ethanolamine kinase 2 \\
\hline 55251 & protein-L-isoaspartate (D-aspartate) O-methyltransferase domain containing 2 \\
\hline 55284 & ubiquitin-conjugating enzyme E2W (putative) \\
\hline 55334 & $\underline{\text { solute carrier family } 39 \text { (zinc transporter), member } 9}$ \\
\hline 55342 & spermatid perinuclear RNA binding protein \\
\hline 55379 & leucine rich repeat containing 59 \\
\hline 55432 & YOD1 OTU deubiquinating enzyme 1 homolog (S. cerevisiae) \\
\hline 55521 & tripartite motif-containing 36 \\
\hline 55619 & dedicator of cytokinesis 10 \\
\hline 55635 & DEP domain containing 1 \\
\hline 55670 & peroxisomal biogenesis factor 26 \\
\hline 55676 & solute carrier family 30 (zinc transporter), member 6 \\
\hline
\end{tabular}




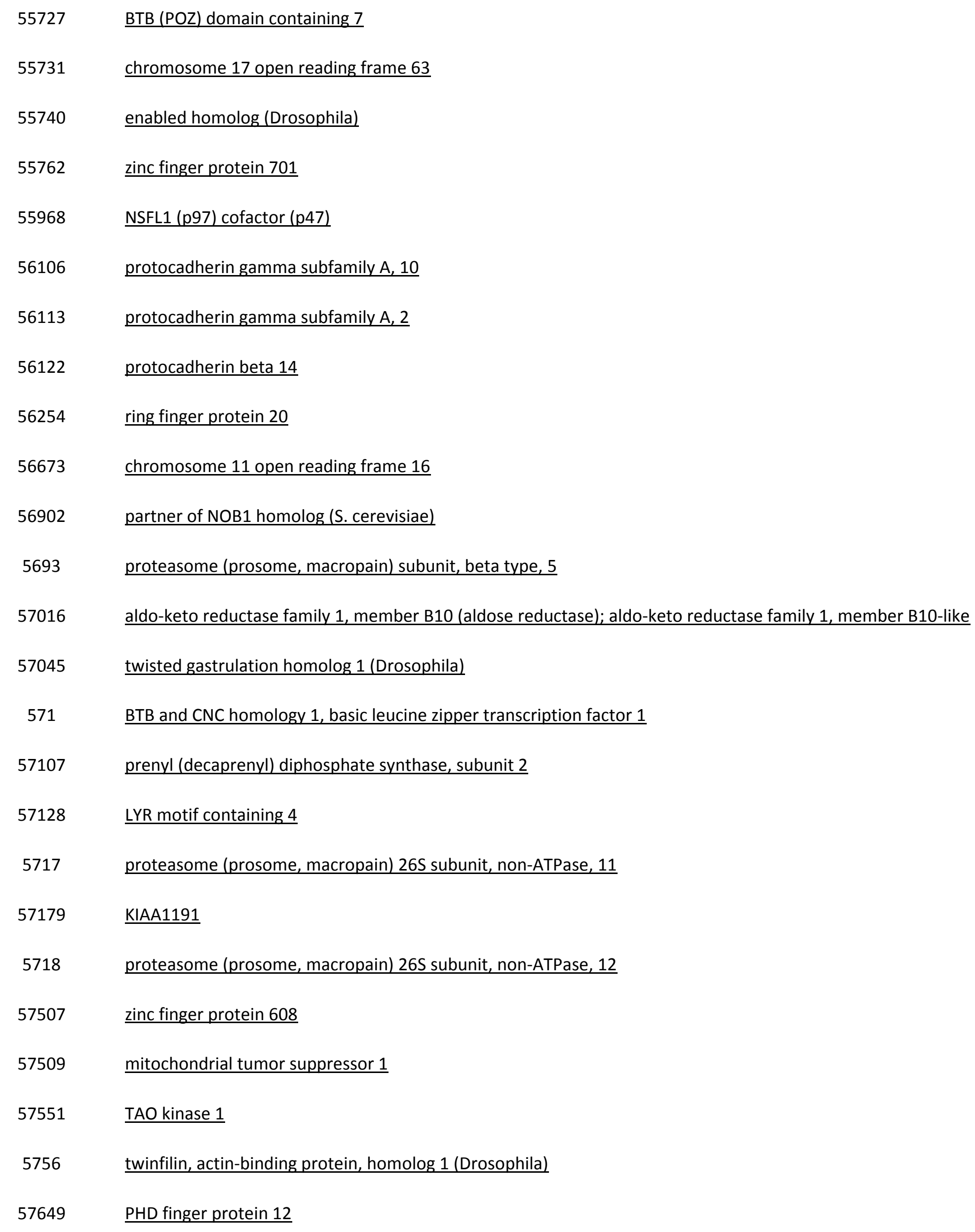




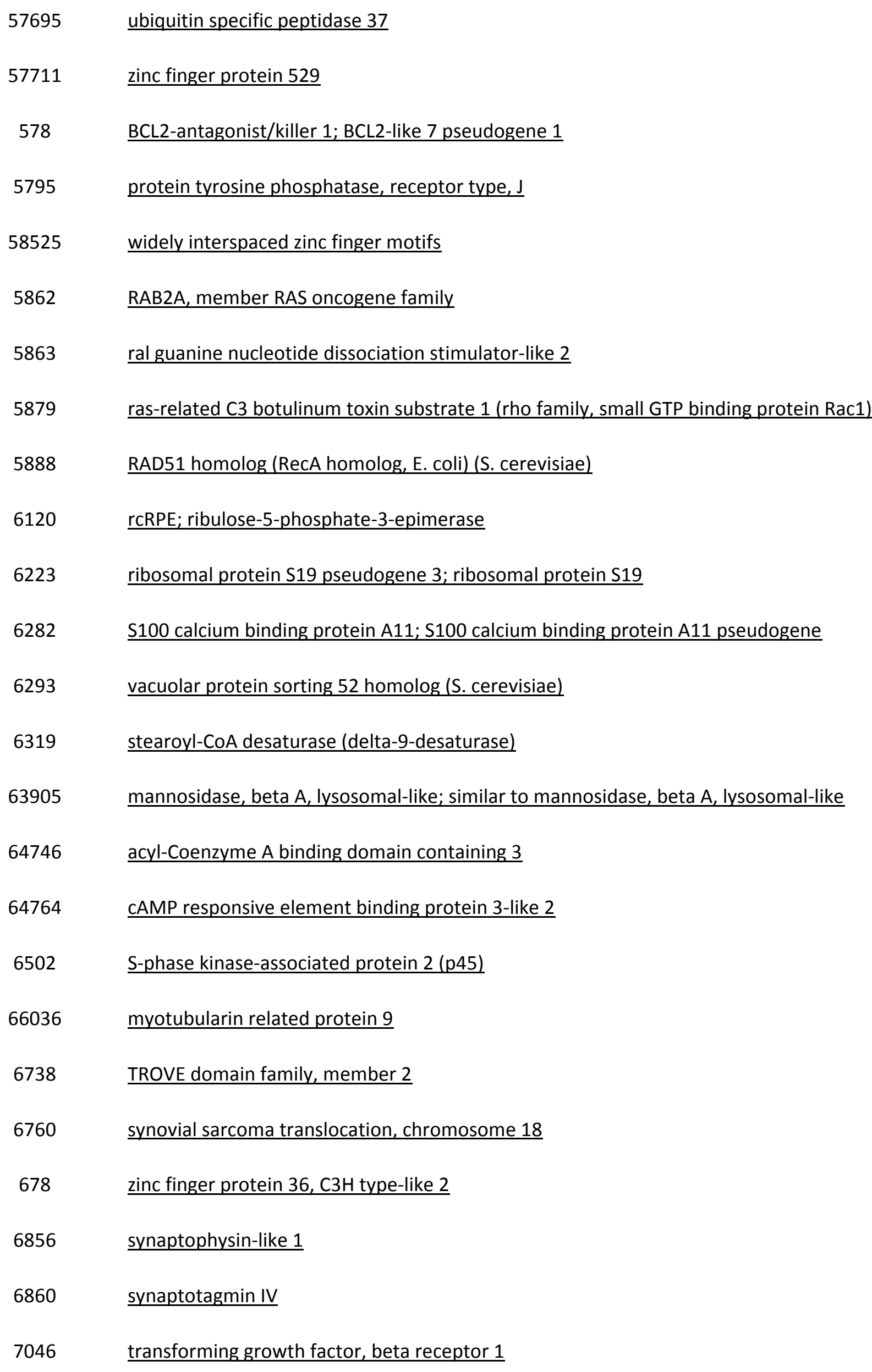




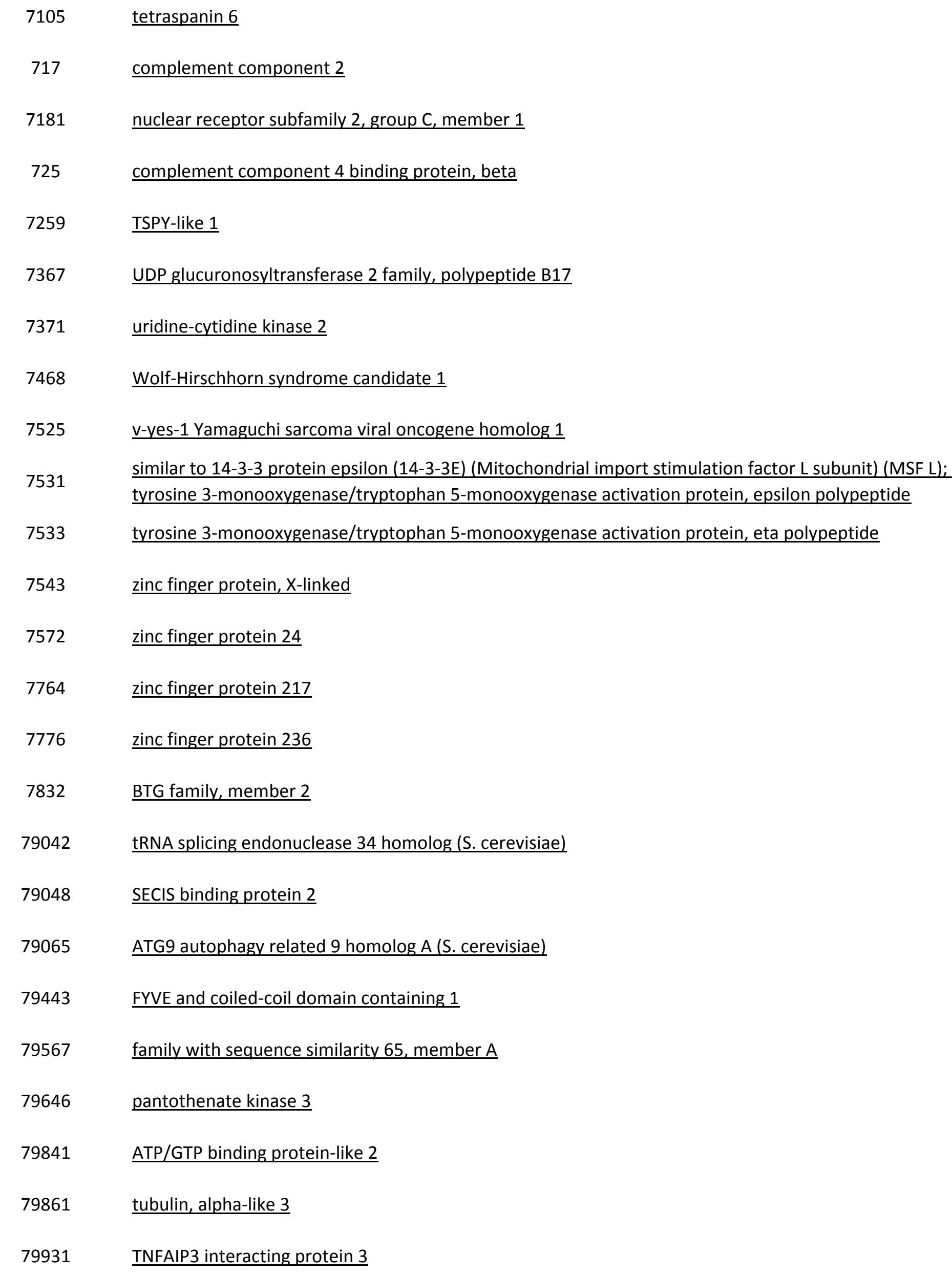


calmodulin 3 (phosphorylase kinase, delta); calmodulin 2 (phosphorylase kinase, delta); calmodulin 1 (phosphorylase kinase, delta)

80255 solute carrier family 35 , member $\mathrm{F5}$

80262 chromosome 16 open reading frame 70

80267 ER degradation enhancer, mannosidase alpha-like 3

8027 signal transducing adaptor molecule (SH3 domain and ITAM motif) 1

$8030 \quad$ coiled-coil domain containing 6

$80311 \quad$ kelch-like 15 (Drosophila)

$8065 \quad$ cullin 5

$8091 \quad$ high mobility group AT-hook 2

81706 protein phosphatase 1, regulatory (inhibitor) subunit $14 \mathrm{C}$

$81790 \quad$ ring finger protein 170

$8324 \quad$ frizzled homolog 7 (Drosophila)

8360 histone cluster 1, H4l; histone cluster 1, H4k; histone cluster 4, H4; histone cluster 1, H4h; histone cluster 1,

H4j; histone cluster 1, H4i; histone cluster 1, H4d; histone cluster 1, H4c; histone cluster 1, H4f; histone cluster

$84224 \quad$ neuroblastoma breakpoint family, member 3

$84255 \quad$ solute carrier family 37 (glycerol-3-phosphate transporter), member 3

84312 breast cancer metastasis-suppressor 1-like

$84335 \quad$ AKT1 substrate 1 (proline-rich)

$84336 \quad$ transmembrane protein 101

$8440 \quad$ NCK adaptor protein 2

$84437 \quad$ KIAA1826

8444 dual-specificity tyrosine-(Y)-phosphorylation regulated kinase 3

$84458 \quad$ ligand dependent nuclear receptor corepressor

$84629 \quad$ trinucleotide repeat containing 18

$84888 \quad$ signal peptide peptidase-like 2A 


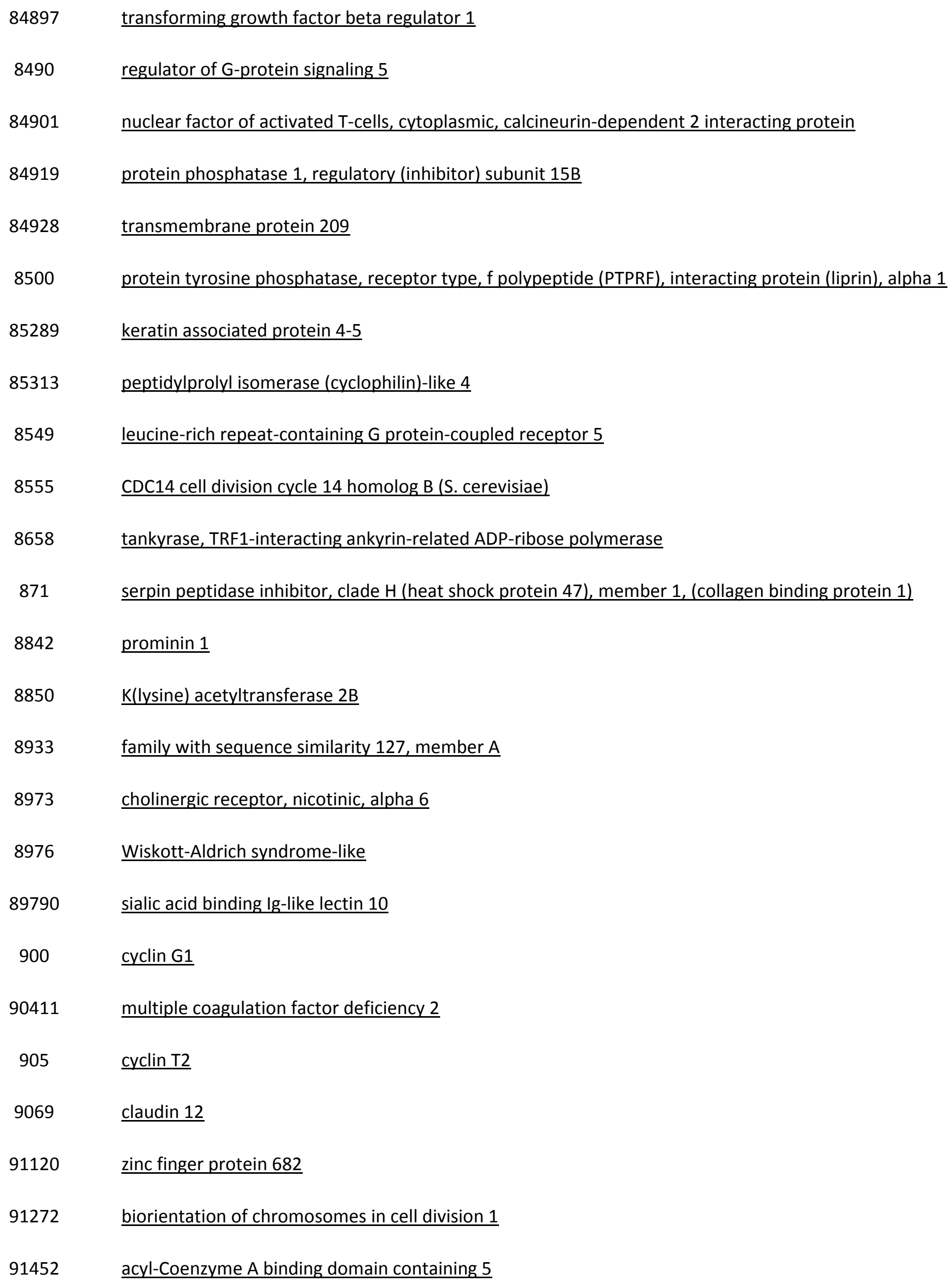




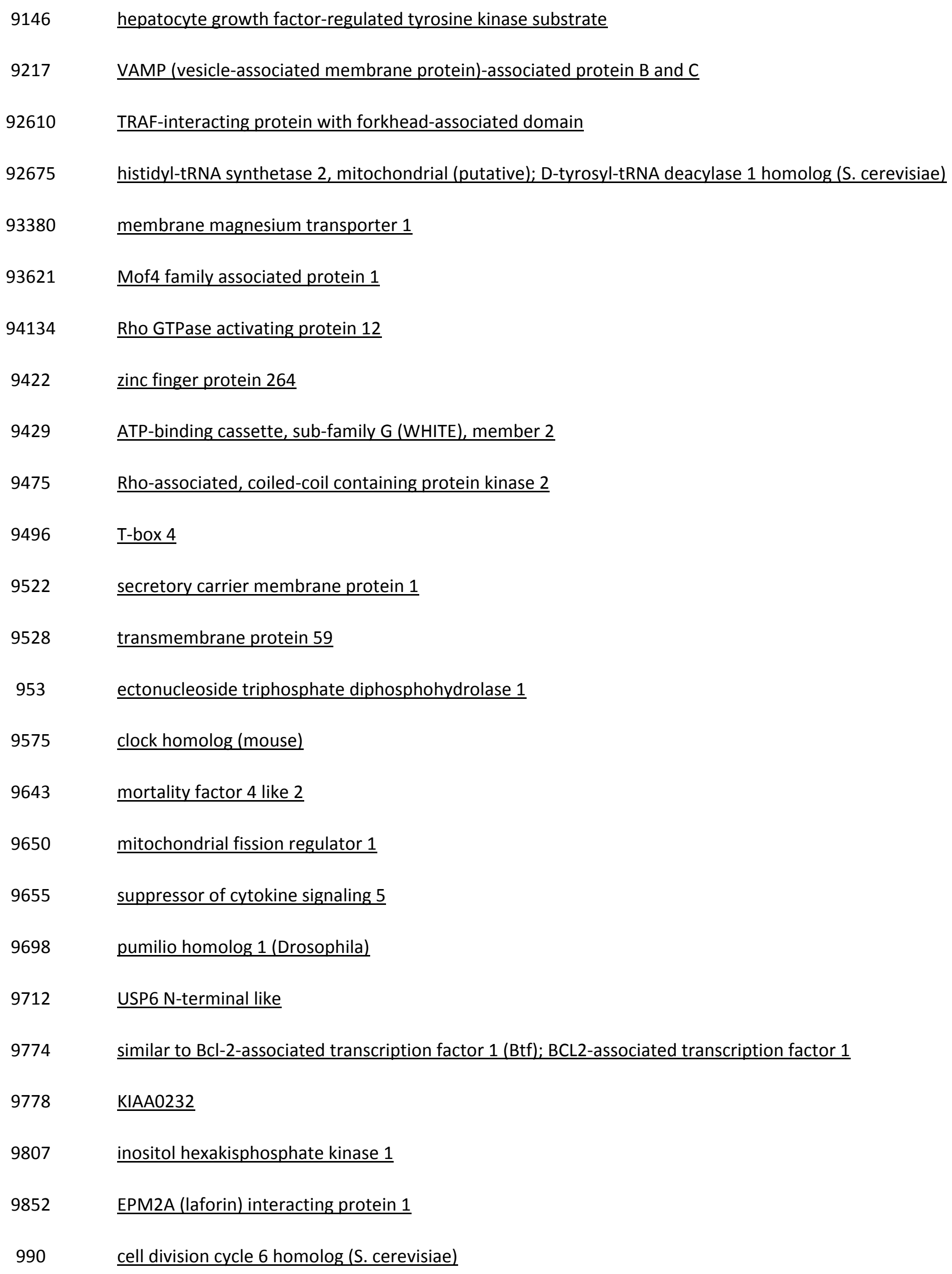


$10135 \quad$ nicotinamide phosphoribosyltransferase

10152 abl interactor 2

10336 polycomb group ring finger 3 
10949 heterogeneous nuclear ribonucleoprotein A0

10951 chromobox homolog 1 (HP1 beta homolog Drosophila)

11145 phospholipase A2, group XVI

11149 blood vessel epicardial substance

$11196 \quad \underline{\text { SEC23 interacting protein }}$

11217 A kinase (PRKA) anchor protein 2; paralemmin 2; PALM2-AKAP2 readthrough transcript

1147 conserved helix-loop-helix ubiquitous kinase

$114757 \quad \underline{\text { cytoglobin }}$

114785 methyl-CpG binding domain protein 6

$114883 \quad$ oxysterol binding protein-like 9

$118987 \quad$ PDZ domain containing 8

\section{9 adducin 2 (beta)}

123879 DCN1, defective in cullin neddylation 1, domain containing 3 (S. cerevisiae)

125 alcohol dehydrogenase 1B (class I), beta polypeptide; alcohol dehydrogenase 1A (class I), alpha polypeptide; alcohol dehydrogenase 1C (class I), gamma polypeptide

126917 intermediate filament family orphan 2

$129684 \quad$ contactin associated protein-like 5

1310 collagen, type XIX, alpha 1

$1316 \quad \underline{\text { Kruppel-like factor } 6}$

$133015 \quad$ PARK2 co-regulated-like

134637 adenosine deaminase, tRNA-specific 2, TAD2 homolog (S. cerevisiae)

139065 SLIT and NTRK-like family, member 4

139285 family with sequence similarity 123B

140456 ankyrin repeat and SOCS box-containing 11 


\begin{tabular}{|c|c|}
\hline 140707 & BRI3 binding protein \\
\hline 142 & poly (ADP-ribose) polymerase 1 \\
\hline 143686 & $\underline{\text { sestrin } 3}$ \\
\hline 144608 & chromosome 12 open reading frame 60 \\
\hline 146760 & reticulon 4 receptor-like 1 \\
\hline 149478 & hypothetical protein LOC149478 \\
\hline 150350 & ENTH domain containing 1 \\
\hline 1510 & cathepsin E \\
\hline 151050 & chromosome 2 open reading frame 67 \\
\hline 151230 & kelch-like 23 (Drosophila) \\
\hline 1528 & cytochrome b5 type A (microsomal) \\
\hline 153222 & chromosome 5 open reading frame 41 \\
\hline 154091 & solute carrier family 2 (facilitated glucose transporter), member 12 \\
\hline 157570 & establishment of cohesion 1 homolog 2 (S. cerevisiae) \\
\hline 160287 & lactate dehydrogenase A-like $6 \mathrm{~A}$ \\
\hline 160418 & $\underline{\text { transmembrane and tetratricopeptide repeat containing } 3}$ \\
\hline 160851 & diacylglycerol kinase, eta \\
\hline 1629 & dihydrolipoamide branched chain transacylase E2 \\
\hline 1657 & Dmx-like 1 \\
\hline 168537 & GTPase, IMAP family member 7 \\
\hline 169200 & $\underline{\text { transmembrane protein } 64}$ \\
\hline 1756 & dystrophin \\
\hline 1831 & TSC22 domain family, member 3; GRAM domain containing 4 \\
\hline 1849 & dual specificity phosphatase 7 \\
\hline 1869 & $\underline{E 2} \mathrm{~F}$ transcription factor 1 \\
\hline
\end{tabular}


$2041 \quad$ EPH receptor A1

\section{EPH receptor A5}

2057 erythropoietin receptor

$205717 \quad$ KIAA2018

2117 ets variant 3

$2120 \quad$ ets variant 6

2150 coagulation factor II (thrombin) receptor-like 1

2152 coagulation factor III (thromboplastin, tissue factor)

2173 fatty acid binding protein 7, brain

$220441 \quad$ ring finger protein 152

$221294 \quad$ 5'-nucleotidase domain containing 1

221981 thrombospondin, type I, domain containing 7A

222662 lipoma HMGIC fusion partner-like 5

$2259 \quad$ fibroblast growth factor 14

2274 four and a half LIM domains 2

22800 related RAS viral (r-ras) oncogene homolog 2; similar to related RAS viral (r-ras) oncogene homolog 2

22874 pleckstrin homology domain containing, family A member 6

22879 MON1 homolog B (yeast)

$22891 \quad$ zinc finger protein 365

2296 forkhead box C1

$23051 \quad$ zinc fingers and homeoboxes 3

$23057 \quad$ nicotinamide nucleotide adenylyltransferase 2

$2308 \quad$ forkhead box 01 
G patch domain containing 8

23136 erythrocyte membrane protein band 4.1-like 3

23236 phospholipase C, beta 1 (phosphoinositide-specific)

23295 mahogunin, ring finger 1

$23321 \quad$ tripartite motif-containing 2

23657 solute carrier family 7, (cationic amino acid transporter, $y+$ system) member 11

$253017 \quad$ steroid 5 alpha-reductase 2-like 2

\section{8 glucose-6-phosphatase, catalytic subunit}

$254048 \quad \underline{\text { ubinuclein } 2}$

254065 bromodomain and WD repeat domain containing 3

254228 family with sequence similarity 26 , member E

256764 WD repeat domain 72

25816 tumor necrosis factor, alpha-induced protein 8

2591 UDP-N-acetyl-alpha-D-galactosamine:polypeptide N-acetylgalactosaminyltransferase 3 (GalNAC-T3)

$25913 \quad$ POT1 protection of telomeres 1 homolog (S. pombe)

$25978 \quad$ chromatin modifying protein 2B

26051 protein phosphatase 1, regulatory (inhibitor) subunit 16B

26071 family with sequence similarity 127, member B

$26147 \quad \underline{\text { PHD finger protein } 19}$

262 adenosylmethionine decarboxylase 1

26524 LATS, large tumor suppressor, homolog 2 (Drosophila)

26973 cysteine and histidine-rich domain (CHORD)-containing 1; cysteine and histidine-rich domain (CHORD)containing 1 pseudogene

$27086 \quad$ forkhead box P1

27109 ATP synthase, $H+$ transporting, mitochondrial FO complex, subunit s (factor B) 
27249 methylmalonic aciduria (cobalamin deficiency) cblD type, with homocystinuria

27287 VENT homeobox homolog (Xenopus laevis)

$2742 \quad$ glycine receptor, alpha 2

283 angiogenin, ribonuclease, RNase A family, 5

283635 family with sequence similarity 177 , member A1

285440 cytochrome P450, family 4, subfamily V, polypeptide 2

$287015 \quad$ tripartite motif-containing 42

2872 MAP kinase interacting serine/threonine kinase 2

\section{$28966 \quad$ sorting nexin 24}

28982 feline leukemia virus subgroup C cellular receptor 1

2904 glutamate receptor, ionotropic, N-methyl D-aspartate 2B

2920 chemokine (C-X-C motif) ligand 2

2965 general transcription factor IIH, polypeptide 1, 62kDa

$29761 \quad$ ubiquitin specific peptidase 25

29843 SUMO1/sentrin specific peptidase 1

$3021 \quad$ H3 histone, family 3B (H3.3B); $\mathrm{H} 3$ histone, family $3 \mathrm{~A}$ pseudogene; $\mathrm{H3}$ histone, family 3A; similar to H3 histone, family $3 \mathrm{~B}$; similar to histone $\mathrm{H} 3.3 \mathrm{~B}$

$30845 \quad$ EH-domain containing 3

$3148 \quad$ high-mobility group box 2

3303 heat shock 70kDa protein 1A; heat shock 70kDa protein 1B

$339230 \quad$ coiled-coil domain containing 137

$3400 \quad$ inhibitor of DNA binding 4, dominant negative helix-loop-helix protein

$340533 \quad \underline{\text { KIAA2022 }}$

341208 hephaestin-like 1 
$344787 \quad$ zinc finger protein 860

3479 insulin-like growth factor 1 (somatomedin C)

$3480 \quad$ insulin-like growth factor 1 receptor

$3569 \quad$ interleukin 6 (interferon, beta 2)

3572 interleukin 6 signal transducer (gp130, oncostatin M receptor)

$3667 \quad$ insulin receptor substrate 1

$367 \quad$ androgen receptor

$3672 \quad$ integrin, alpha 1

3688 integrin, beta 1 (fibronectin receptor, beta polypeptide, antigen CD29 includes MDF2, MSK12)

3760 potassium inwardly-rectifying channel, subfamily J, member 3

$387032 \quad$ zinc finger with KRAB and SCAN domains 4

\section{$388 \quad$ ras homolog gene family, member B}

389072 pleckstrin homology domain containing, family M, member 3

$3925 \quad$ stathmin 1

$3949 \quad$ low density lipoprotein receptor

$3976 \quad$ leukemia inhibitory factor (cholinergic differentiation factor)

$3977 \quad$ leukemia inhibitory factor receptor alpha

4005 LIM domain only 2 (rhombotin-like 1)

4026 LIM domain containing preferred translocation partner in lipoma

$402665 \quad$ IgLON family member 5

$4076 \quad$ cell cycle associated protein 1

$408 \quad$ arrestin, beta 1

$4084 \quad$ MAX dimerization protein 1 
muskelin 1, intracellular mediator containing kelch motifs

$4300 \quad$ myeloid/lymphoid or mixed-lineage leukemia (trithorax homolog, Drosophila); translocated to, 3

445815 A kinase (PRKA) anchor protein 2; paralemmin 2; PALM2-AKAP2 readthrough transcript

$4685 \quad$ neural cell adhesion molecule 2

472 similar to Serine-protein kinase ATM (Ataxia telangiectasia mutated) (A-T, mutated); ataxia telangiectasia mutated

$4735 \quad$ septin 2

$4753 \quad$ NEL-like 2 (chicken)

$4774 \quad$ nuclear factor I/A

$4782 \quad$ nuclear factor I/C (CCAAT-binding transcription factor)

$4784 \quad$ nuclear factor I/X (CCAAT-binding transcription factor)

$4858 \quad$ neuro-oncological ventral antigen 2

$488 \quad$ ATPase, Ca++ transporting, cardiac muscle, slow twitch 2

$4899 \quad$ nuclear respiratory factor 1

$5013 \quad$ orthodenticle homeobox 1

$5078 \quad$ paired box 4

5080 paired box 6

$51022 \quad$ glutaredoxin 2

$51141 \quad$ insulin induced gene 2

51232 cysteine rich transmembrane BMP regulator 1 (chordin-like)

51393 transient receptor potential cation channel, subfamily V, member 2

$51533 \quad$ PHD finger protein 7 
$5216 \quad$ profilin 1

5243 ATP-binding cassette, sub-family B (MDR/TAP), member 1

5324 pleiomorphic adenoma gene 1

$53344 \quad$ cysteine-rich hydrophobic domain 1

$5354 \quad$ proteolipid protein 1

5366 phorbol-12-myristate-13-acetate-induced protein 1

$538 \quad$ ATPase, $\mathrm{Cu}++$ transporting, alpha polypeptide

$54328 \quad$ G protein-coupled receptor 173

$54462 \quad \underline{\text { KIAA1128 }}$

$54516 \quad$ mitochondrial translational release factor 1-like

$54558 \quad$ spermatogenesis associated 6

$54602 \quad$ Nedd4 family interacting protein 2

$54664 \quad$ transmembrane protein 106B

$54796 \quad$ basonuclin 2

$54808 \quad$ dymeclin

$5481 \quad$ peptidylprolyl isomerase D

$54811 \quad$ zinc finger protein 562

$54978 \quad$ chromosome 2 open reading frame 18

5501 protein phosphatase 1, catalytic subunit, gamma isoform

$55071 \quad$ chromosome 9 open reading frame 40

$55183 \quad$ RAP1 interacting factor homolog (yeast)

$55207 \quad$ ADP-ribosylation factor-like 8B

$55294 \quad$ F-box and WD repeat domain containing 7

55508 solute carrier family 35 , member E3

55667 DENN/MADD domain containing 4C 


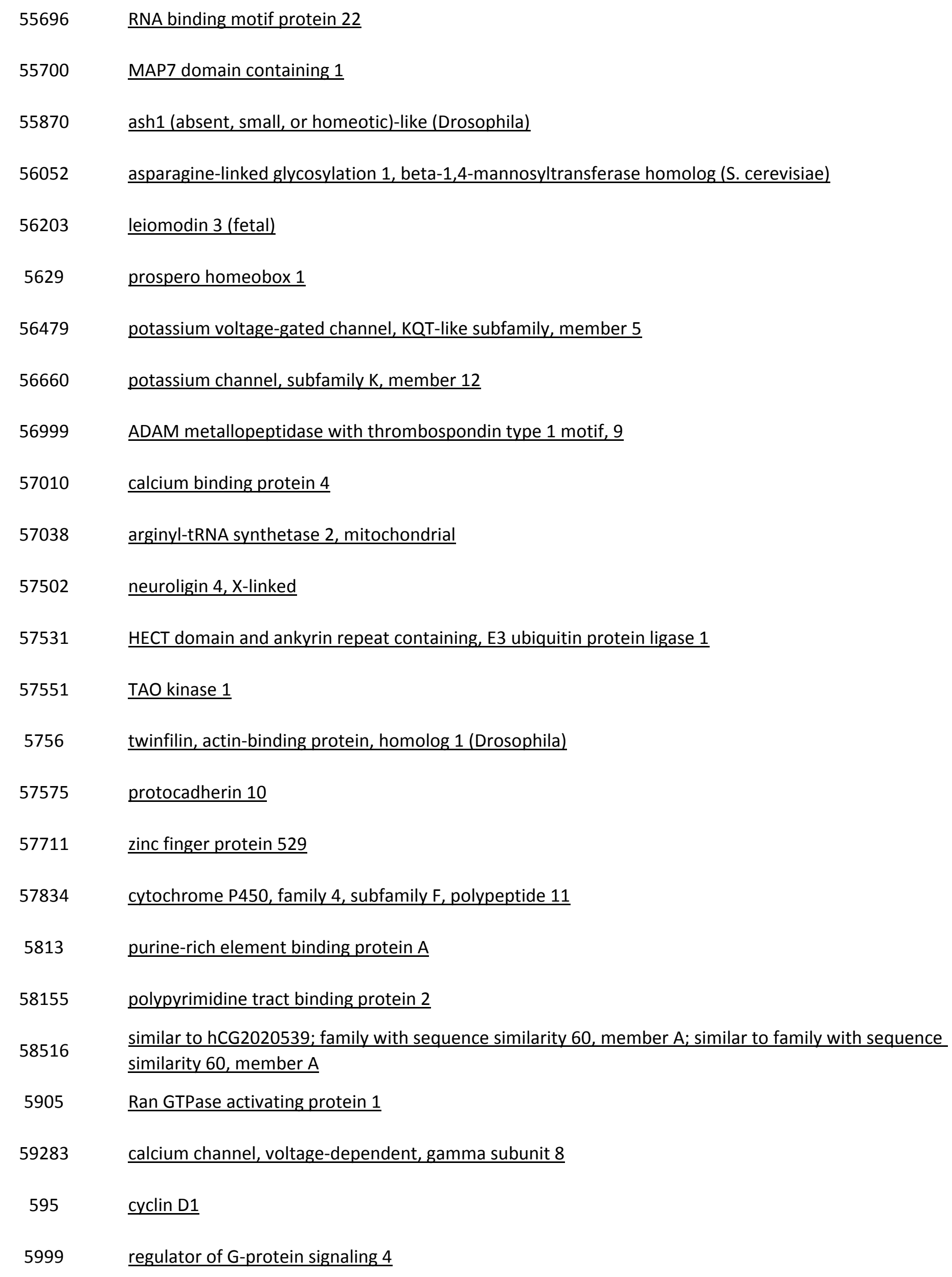


SATB homeobox 1

sterol-C4-methyl oxidase-like

6348 chemokine (C-C motif) ligand 3

$63899 \quad$ NOL1/NOP2/Sun domain family, member 3

PR domain containing 1, with ZNF domain

$64121 \quad$ Ras-related GTP binding C

$64168 \quad \mathrm{~N}$-terminal EF-hand calcium binding protein 1

$64399 \quad$ hedgehog interacting protein

6469 sonic hedgehog homolog (Drosophila)

6517 solute carrier family 2 (facilitated glucose transporter), member 4

657 bone morphogenetic protein receptor, type IA; similar to ALK-3

65977 pleckstrin homology domain containing, family A (phosphoinositide binding specific) member 3

6602 SWI/SNF related, matrix associated, actin dependent regulator of chromatin, subfamily $d$, member 1

$6643 \quad$ sorting nexin 2

$6660 \quad \underline{\operatorname{SRY}(\text { sex determining region Y)-box } 5}$

$6667 \quad$ Sp1 transcription factor

$6670 \quad$ Sp3 transcription factor

$6776 \quad$ signal transducer and activator of transcription $5 \mathrm{~A}$

$6886 \quad$ T-cell acute lymphocytic leukemia 1

$7157 \quad$ tumor protein $\mathrm{p} 53$

7184 heat shock protein 90kDa beta (Grp94), member 1

$7188 \quad$ TNF receptor-associated factor 5

727936 glycosyltransferase 8 domain containing 4 
$7517 \quad$ X-ray repair complementing defective repair in Chinese hamster cells 3

$7693 \quad$ zinc finger protein 134

$79027 \quad$ zinc finger protein 655

$79184 \quad$ BRCA1/BRCA2-containing complex, subunit 3

79733 E2F transcription factor 8

$79782 \quad$ leucine rich repeat containing 31

$79834 \quad$ NKF3 kinase family member

79956 endoplasmic reticulum metallopeptidase 1

79991 oligonucleotide/oligosaccharide-binding fold containing 1

80018 chromosome 12 open reading frame 30

$80034 \quad$ cysteine-serine-rich nuclear protein 3

80167 chromosome 4 open reading frame 29

80311 kelch-like 15 (Drosophila)

$8078 \quad$ ubiquitin specific peptidase 5 (isopeptidase T)

8082 sarcospan (Kras oncogene-associated gene)

$80830 \quad$ apolipoprotein L, 6

$8140 \quad$ solute carrier family 7 (cationic amino acid transporter, $y+$ system), member 5

81848 sprouty homolog 4 (Drosophila)

$8208 \quad$ chromatin assembly factor 1 , subunit B ( $\mathrm{p} 60$ )

83259 protocadherin 11 Y-linked

$83604 \quad \underline{\text { transmembrane protein } 47}$

$83935 \quad$ transmembrane protein 133

$84056 \quad$ katanin $\mathrm{p} 60$ subunit A-like 1 


\section{$84437 \quad \underline{\text { KIAA1826 }}$}

84529 chromosome 15 open reading frame 41

84552 par-6 partitioning defective 6 homolog gamma (C. elegans)

$84826 \quad$ SFT2 domain containing 3

$84888 \quad$ signal peptide peptidase-like 2A

84898 plexin domain containing 2

$8490 \quad$ regulator of G-protein signaling 5

84945 abhydrolase domain containing 13

$85379 \quad$ KIAA1671 protein

$85440 \quad$ dedicator of cytokinesis 7

85446 zinc finger homeobox 2

862 runt-related transcription factor 1; translocated to, 1 (cyclin D-related)

$8674 \quad$ vesicle-associated membrane protein 4

8933 family with sequence similarity 127 , member A

$8976 \quad$ Wiskott-Aldrich syndrome-like

$90141 \quad$ chromosome 14 open reading frame 143

$9048 \quad$ artemin

$9060 \quad 3$ '-phosphoadenosine 5'-phosphosulfate synthase 2

$90624 \quad$ Lyrm7 homolog (mouse)

$91147 \quad$ transmembrane protein 67

91452 acyl-Coenzyme A binding domain containing 5

$91807 \quad$ myosin light chain kinase 3

$91860 \quad$ calmodulin-like 4

9255 aminoacyl tRNA synthetase complex-interacting multifunctional protein 1

93034 retinol dehydrogenase 14 (all-trans/9-cis/11-cis); 5'-nucleotidase, cytosolic IB 
BCL2-associated athanogene 2

$957 \quad$ ectonucleoside triphosphate diphosphohydrolase 5

\section{$96459 \quad$ folliculin interacting protein 1}

$9727 \quad$ RAB11 family interacting protein 3 (class II)

$9728 \quad$ SECIS binding protein 2-like

$9731 \quad$ KIAA0562

$9754 \quad$ StAR-related lipid transfer (START) domain containing 8

$9760 \quad$ thymocyte selection-associated high mobility group box

$989 \quad \underline{\operatorname{septin} 7}$

$9899 \quad$ synaptic vesicle glycoprotein 2B; hypothetical protein LOC100128403 


\section{GENE NAME}

zinc finger protein 460

methyl-CpG binding domain protein 6

$\underline{\text { transmembrane and tetratricopeptide repeat containing } 3}$

epithelial cell transforming sequence 2 oncogene

\section{MON1 homolog B (yeast)}

solute carrier family 7, (cationic amino acid transporter, $y+$ system) member 11

family with sequence similarity 127 , member $B$

family with sequence similarity 177 , member $A 1$

feline leukemia virus subgroup C cellular receptor 1

interleukin 6 (interferon, beta 2 )

interleukin 6 signal transducer (gp130, oncostatin M receptor)

LIM domain containing preferred translocation partner in lipoma

muskelin 1, intracellular mediator containing kelch motifs

ATPase, Ca++ transporting, cardiac muscle, slow twitch 2

insulin induced gene 2

chromosome 2 open reading frame 18

TAO kinase 1

twinfilin, actin-binding protein, homolog 1 (Drosophila)

zinc finger protein 529

kelch-like 15 (Drosophila)

$\underline{\text { KIAA1826 }}$

signal peptide peptidase-like $2 \mathrm{~A}$

regulator of G-protein signaling 5 
family with sequence similarity 127 , member A

Wiskott-Aldrich syndrome-like

acyl-Coenzyme A binding domain containing 5 